\title{
A low dimensional fluid motion estimator
}

\author{
Anne Cuzol Pierre Hellier Etienne Mémin
}

\author{
IRISA, Université de Rennes 1 \\ Campus de Beaulieu \\ 35042 Rennes Cedex, France \\ \{acuzol,phellier,memin\}@irisa.fr
}

\begin{abstract}
In this paper we propose a new motion estimator for image sequences depicting fluid flows. The proposed estimator is based on the Helmholtz decomposition of vector fields. This decomposition consists in representing the velocity field as a sum of a divergence free component and a vorticity free component. The objective is to provide a low-dimensional parametric representation of optical flows by depicting them as deformations generated by a reduced number of vortex and source particles. Both components are approximated using a discretization of the vorticity and divergence maps through regularized Dirac measures. The resulting so called irrotational and solenoidal fields consist of linear combinations of basis functions obtained through a convolution product of the Green kernel gradient and the vorticity map or the divergence map respectively. The coefficient values and the basis function parameters are obtained by minimization of a functional relying on an integrated version of mass conservation principle of fluid mechanics. Results are provided on synthetic examples and real world sequences.
\end{abstract}

\section{Introduction}

The observation, understanding and control of complex fluid flows is a major scientific issue. For instance, in environmental sciences such as oceanography, meteorology and climatology, the monitoring and the forecasting of the atmosphere or the ocean is becoming more and more crucial for our everyday life. The physical understanding of these flows is poorly known because of (a) their complex nature and (b) unknown or inaccurate border conditions. Accurate and dense measurements can hardly be recovered by probes or by numerical evaluation of current physical 
models. In that prospect, imaging sensors are very attractive as they provide multi-modal data at high spatio-temporal resolution. Besides, we can point out the fact that in numerous domains of applications imaging sensors are almost the only practical means to get some information on the flow of interest. This is the case for instance in meteorology where some parts of the globe are not well covered by meteorological stations. This is all the more problematic when one aims at studying global atmospheric circulation or climate evolution. The same situation appears for large scale experiments in fluid mechanics or for blood flow analysis, as in situ measurements are difficult.

The analysis of dynamic structures and the estimation of velocities for fluid image sequences have received great attention from the computer vision community for several years [19, 21, 28, 34, 43, 45]. Application domains range from experimental visualization in fluid mechanics, environmental sciences (oceanography, meteorology, ...), to medical imagery. Recently, several dedicated approaches have been proposed for fluid flow velocity estimation [13, 26, 44]. Unlike most of the motion estimators based on the brightness consistency assumption and a first order smoothness function, these techniques rely on a data-model derived from the continuity equation of fluid mechanics and second order div-curl regularizers. A first order regularizer (eventually associated to a robust cost function) favors piecewise translational motion fields by penalizing high gradients of the solution. In the same way, second order div-curl penalizers encourage solutions with blobs of piecewise constant divergence and curl. These methods are conceptually much more satisfying as they comply with the brightness variations and the motion observed in fluid image sequences. However, these estimators are dense estimators and the solutions associated belong to spaces of great dimension. It is desirable for some applications such as tracking to provide low dimensional solutions. This is the purpose of this paper.

Such a low dimensional description of fluid flows allows a characterization of meaningful areas of the flow in terms of vorticity and divergence. These areas are the centers of kinematical events of great interest to describe the observed flow. In this document, we propose a technique to estimate a low dimensional representation of fluid motion fields. This method relies on the Helmholtz decomposition of a motion field (also used by other fluid flow analysis methods $[14,26,44])$ which consists in decoupling the vector field into a divergence free component and a vorticity free component. The method is based on a discrete representation of the vorticity (also called curl) and divergence maps. This discretization enables to define implicitly adapted regularizers for fluid motion estimation problems. Such a technique is related to motion estimators based on radial basis functions [3]. Nevertheless, contrary to techniques that operate directly a regularized discretization of the motion field, the proposed method relies on the dis- 
cretization of the curl and the divergence of the motion field. It provides a simplified description of the vorticity and divergence maps of a flow observed through an image sequence. It enables therefore to provide at the end a kind of motion segmentation map. The fact that this estimator provides as a by-product a simplified vorticity/divergence map of the flow is also a very interesting point of the method we propose. As a matter of fact, this latter point allows to provide a reduced system of descriptors to analyse the main characteristics of the flow.

The paper is organized as follows. After a review of the properties of vector fields in section 2 and a presentation of the parametric model we use in section 3, we present in section 4 a way to construct the fluid motion estimation problem from such a modelization. The estimation method of the low dimensional representation is then further described. The proposition of a fluid motion estimator based on such a parametric representation constitutes the main contribution of this paper. Section 6 shows results for synthetic and real examples. Results on fluid motion analysis are shown for applications in meteorology and fluid mechanics. Results on medical images are also presented.

This paper is an extended version of [16] and [18], respectively dedicated to the estimation of fluid motions and to a non-rigid registration problem in medical imaging.

\section{Definitions and properties of vector fields}

In this section, known analytic results on planar vector fields are recalled. We shall rely on them to develop an original method for fluid motion estimation.

A two-dimensional vector field $\mathbf{w}$ is a $\mathbb{R}^{2}$-valued map defined on a bounded set $\Omega$ of $\mathbb{R}^{2}$. We denote it $\mathbf{w}(\mathbf{x})=(u(\mathbf{x}), v(\mathbf{x}))^{T}$, where $\mathbf{x}=(x, y)$ and $x$ and $y$ are the spatial coordinates. Each component of the vector field will be supposed twice continuously differentiable: $u, v \in$ $\mathcal{C}^{2}(\Omega, \mathbb{R})$.

Noting $\boldsymbol{\nabla}=\left(\frac{\partial}{\partial x}, \frac{\partial}{\partial y}\right)$ the operator whose components are the partial derivatives with respect to the coordinates $x$ and $y$, we define the divergence and the scalar vorticity of the vector field:

$$
\left\{\begin{array}{l}
\operatorname{div} \mathbf{w}=\frac{\partial u}{\partial x}+\frac{\partial v}{\partial y}=\nabla \cdot \mathbf{w}, \\
\operatorname{curl} \mathbf{w}=\frac{\partial u}{\partial y}-\frac{\partial v}{\partial x}=\nabla \cdot \mathbf{w}^{\perp}
\end{array}\right.
$$

where $\mathbf{w}^{\perp}=(-v, u)$ is the orthogonal counterpart of $\mathbf{w}$.

The vorticity accounts for the presence of a rotating motion, while the divergence is related to the presence of sinks or sources in the flow. A vector field whose divergence is null at every 
point is called solenoidal. Similarly, a field with zero vorticity will be called irrotational. It is well known that for irrotational fields there exists a scalar function $\phi$, called the velocity potential, such that $\mathbf{w}=\boldsymbol{\nabla} \phi$. Similarly, for solenoidal fields (whose orthogonal component is an irrotational field) there exists a scalar function $\psi$ called the stream function such that $\mathbf{w}^{\perp}=\nabla \psi$.

Any continuous vector field that vanishes at infinity can be decomposed into a sum of an irrotational component with null vorticity and a solenoidal component with null divergence. This is called the Helmholtz decomposition: $\mathbf{w}=\mathbf{w}_{\text {irr }}+\mathbf{w}_{\text {sol }}$. When the null border condition can not be imposed, an additional component $\mathbf{w}_{\text {tra }}$, named transportation component, which is both irrotational and solenoidal, has to be included. The decomposition reads then: $\mathbf{w}=$ $\mathbf{w}_{\text {irr }}+\mathbf{w}_{\text {sol }}+\mathbf{w}_{\text {tra }}$. This last component can be approximated using the Horn and Schunck estimator with a strong regularization coefficient [14]. In the sequel, we will assume that the transportation component has been previously computed and that its associated motion has been removed from the image sequence. We will consequently assume a null border condition at infinity knowing that the image sequence, $I(\mathbf{x}, t)$, is related to the original image sequence, $I_{o}(\mathbf{x}, t)$, by $I(\mathbf{x}, t)=I_{o}\left(\mathbf{x}+\mathbf{w}_{\text {tra }}(\mathbf{x}, t), t\right)$.

Substituting the two components $\mathbf{w}_{i r r}$ and $\mathbf{w}_{\text {sol }}$ by their expressions in terms of potential functions, the Helmholtz decomposition reads:

$$
\mathbf{w}=\nabla \phi+\nabla^{\perp} \psi
$$

Considering the divergence and the curl of the motion field yields to two so-called Poisson equations:

$$
\Delta \phi=\operatorname{div} \mathbf{w} \quad \text { and } \quad \Delta \psi=-\operatorname{curl} \mathbf{w}
$$

where $\Delta$ denotes the Laplacian operator and whose solutions are the potential functions $\phi$ and $\psi$. These solutions may be expressed as convolution products:

$$
\begin{gathered}
\phi(\mathbf{x})=\int G(\mathbf{x}-\mathbf{u}) \operatorname{div} \mathbf{w}(\mathbf{u}) d \mathbf{u}=G * \operatorname{div} \mathbf{w}(\mathbf{x}), \\
\psi(\mathbf{x})=-\int G(\mathbf{x}-\mathbf{u}) \operatorname{curl} \mathbf{w}(\mathbf{u}) d \mathbf{u}=-G * \operatorname{curl} \mathbf{w}(\mathbf{x}),
\end{gathered}
$$

where $G$ is the Green's function associated to the two-dimensional Laplacian:

$$
G(\mathbf{x})=\frac{1}{2 \pi} \ln (|\mathbf{x}|) .
$$


As the vector fields $\mathbf{w}_{i r r}$ and $\mathbf{w}_{\text {sol }}$ are respectively the gradient and the orthogonal gradient of the potential functions $\phi$ and $\psi$, equations (4) and (5) may be rewritten as:

$$
\mathbf{w}_{\text {irr }}(\mathbf{x})=K * \operatorname{div} \mathbf{w}(\mathbf{x}) \quad \text { and } \quad \mathbf{w}_{\text {sol }}(\mathbf{x})=-K^{\perp} * \operatorname{curl} \mathbf{w}(\mathbf{x})
$$

where $K$ denotes the gradient of the Green kernel:

$$
K(\mathbf{x})=\frac{\mathbf{x}}{2 \pi|\mathbf{x}|^{2}}
$$

The second equation of (7) is known as the Biot-Savart integral. These two equations state that the solenoidal and the irrotational components (and consequently the whole vector field) may be recovered through a convolution product knowing the vorticity and the divergence of the velocity field.

\section{Vortex particles}

The idea of vortex particles methods $[9,29]$ consists in representing the vorticity distribution of a field by a set of discrete amounts of vorticity (vortices). Using these vortices, the vorticity distribution is mathematically approximated by:

$$
\operatorname{curl} \mathbf{w}(\mathbf{x}) \approx \sum_{i} \gamma_{i} \delta\left(\mathbf{x}-\mathbf{z}_{i}\right)
$$

where $\mathbf{z}_{i}$ is the location of the vortex $i, \gamma_{i}$ the strength of the vortex and $\delta$ the Dirac delta function. The vortices are called point vortices since they are represented by delta functions.

The discretization of the vorticity into a limited number of elements enables to evaluate the velocity field directly from the Biot-Savart integral (equ. 7). However, because of the singularity of the Green kernel gradient $K$, the velocity field becomes unbounded if any two vortices come very close to each other. In fact, the induced field develops $\frac{1}{r}$-type singularities, where $r$ is the distance to the point vortices. These singularities can be removed by smoothing the Dirac measure with a cutt-off or blob function, leading to a smoothed version of $K$ and to new methods called vortex blob methods. A vortex blob is obtained by spreading the vorticity of a point vortex over a small chosen area. The blob function describes the vorticity distribution in this area.

There are many ways to define the blob function. A trade off between smoothing and estimation accuracy must be found. Let $f_{\epsilon}$ be such a blob function scaled by a parameter $\epsilon$ : $f_{\epsilon}(\mathbf{x})=\frac{1}{\epsilon^{2}} f\left(\frac{\mathbf{x}}{\epsilon}\right)$. The smoothed kernel is defined as $K_{\epsilon}=K * f_{\epsilon}$. The amount of smoothing is 
determined by the value of $\epsilon$. If $\epsilon$ goes to zero, $f_{\epsilon}$ tends to the Dirac function and $K_{\epsilon}$ tends to $K$. The error induced by the smoothing can be reduced by choosing a function with some moment properties. In fact, if the blob function satisfies:

$$
\begin{aligned}
& \int f(\mathbf{x}) d \mathbf{x}=1, \\
& \int \mathbf{x}^{i} f(\mathbf{x}) d \mathbf{x}=0 \quad i \leq r-1, \\
& \int|\mathbf{x}|^{r}|f(\mathbf{x})| d \mathbf{x}<\infty
\end{aligned}
$$

its order is $r$ and it has the same moment properties as the Dirac measure, up to the power $r-1$.

A starting point to construct a smoothing function is to choose a radially symmetric function $f$ with infinite support, whose mass is essentially concentrated on a disc of radius $\epsilon$. Then, for some proper choices of $f$, the property of radial symmetry leads to an explicit form for $K_{\epsilon}$. In fact we can write [15]:

$$
K_{\epsilon}(\mathbf{x})=\frac{\mathbf{x}}{|\mathbf{x}|^{2}} \int_{0}^{|\mathbf{x}|} \mathbf{s} f_{\epsilon}(\mathbf{s}) d \mathbf{s},
$$

and consequently, if an analytic expression of the integral is available, the entire expression for $K_{\epsilon}$ is known.

Rectangle basis function and Rankine vortex model As a first example let us consider a centered radial rectangle basis function defined as:

$$
f_{\epsilon}(\mathbf{x})=\frac{1}{2 \pi \epsilon^{2}} \mathbb{I}_{\|\mathbf{x}\| \leq \epsilon},
$$

where the function $\mathbb{1}_{\|\mathbf{x}\| \leq \epsilon}=1$ if $\|\mathbf{x}\| \leq \epsilon$ and is null otherwise. The expression of the associated centered smoothed kernel $K_{\epsilon}$, associated to a constant vorticity of strength $\alpha$ inside the disc of radius $\epsilon$ (respectively a divergence of strength $\beta$ ), leads to the well known Rankine vortex model [10]. The velocity for such a vortex model is then defined as:

$$
\begin{aligned}
\mathbf{w}_{\text {sol }}(\mathbf{x}) & =-K^{\perp} * \alpha \mathrm{I}_{\|\mathbf{x}\| \leq \epsilon} \\
& =-\frac{\mathbf{x}^{\perp} \alpha}{2 \pi \epsilon^{2}} 1 \mathbf{I}_{\| \mathbf{x} \mid \leq \epsilon}-\frac{\mathbf{x}^{\perp} \alpha}{2 \pi\|\mathbf{x}\|^{2}} \mathbb{I}_{\| \mathbf{x} \mid>\epsilon} .
\end{aligned}
$$

This model which states that the velocity is linear inside a disc of radius $\epsilon$ and decreases in $1 /\|\mathbf{x}\|^{2}$ outside of the circular domain has been already used in image analysis studies $[14,34,36]$. That kind of model has been used to segment fluid motion fields in oder to characterize vortex or sink/source areas. Such a simple radial basis function can be used when centered 
on critical points of the velocity field. The estimation of the critical points of the velocity field requires nevertheless the complete knowledge of the motion field. The motion field being known the parameters of the Rankine model can be estimated through an iterative process [14]. Otherwise, techniques based on alternating dense motion estimation and Rankine's model parameters estimation must be settled [34].

Gaussian smoothing function A Gaussian function constitutes a trade off between a convenient analytical use and the smoothness properties of the corresponding smoothed kernel (a Gaussian is of second order following the classification given above). The choice of the Gaussian function $f(\mathbf{x})=\frac{1}{\pi} \exp \left(-|\mathbf{x}|^{2}\right)$ leads to the following expression for the smoothed kernel $K_{\epsilon}$ :

$$
K_{\epsilon}(\mathbf{x})=\frac{\mathbf{x}}{2 \pi|\mathbf{x}|^{2}}\left(1-\exp \left(-\frac{|\mathbf{x}|^{2}}{\epsilon^{2}}\right)\right) .
$$

Figure 1 shows the effect of the smoothing by this Gaussian cutt-off and illustrates the fact that $K_{\epsilon} \rightarrow K$ as $\epsilon \rightarrow 0$.

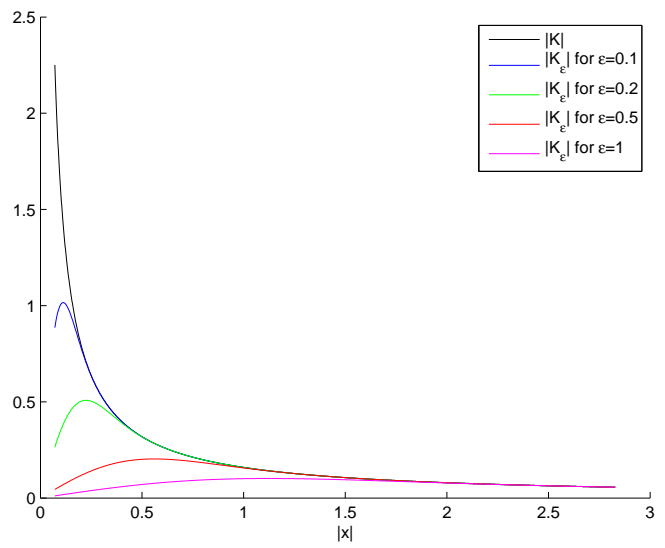

Figure 1: Comparison between the modulus of the original kernel $K$ and the Gaussian-smoothed version $K_{\epsilon}$ for some values of $\epsilon$.

The vorticity distribution of the field $\mathbf{w}$ is finally represented by a linear combination of basis functions called vortex particles:

$$
\operatorname{curl} \mathbf{w}(\mathbf{x}) \approx \sum_{i} \gamma_{i} f_{\epsilon_{i}}\left(\mathbf{x}-\mathbf{z}_{i}\right)
$$


The same idea can be applied to equation (4). This leads to a representation of the divergence distribution in term of a linear combination of functions that we call source particles. For the divergence map, the smoothed source particles representation then reads:

$$
\operatorname{div} \mathbf{w}(\mathbf{x}) \approx \sum_{i} \gamma_{i} f_{\epsilon_{i}}\left(\mathbf{x}-\mathbf{z}_{i}\right)
$$

where $\mathbf{z}_{i}$ denotes the center of each basis function $f_{\epsilon_{i}}$, the coefficient $\gamma_{i}$ is the strength associated to the particle $i$, and $\epsilon_{i}$ represents its influence domain. These parameters are free to vary from a function to another.

\section{Fluid motion estimation from image sequences}

In this section, we present how a vortex and source particles representation may be used in conjunction with an appropriate cost function to devise a motion estimator for image sequences depicting fluid flows.

\subsection{Motion representation}

As presented above, discretizing the vorticity map with $p$ vortex particles associated with a Gaussian smoothing of the Dirac measure enables to recover the solenoidal component. The Biot-Savart integration leads to the following analytic representation of the solenoidal motion field:

$$
\mathbf{w}_{\text {sol }}(\mathbf{x}) \approx \sum_{i=0}^{p} \gamma_{i}^{\text {sol }} K^{\perp} * f_{\epsilon_{i}^{s o l}}\left(\mathbf{z}_{i}^{\text {sol }}-\mathbf{x}\right)=\sum_{i=0}^{p} \gamma_{i}^{\text {sol }} K_{\epsilon_{i}^{\text {sol }}}^{\perp}\left(\mathbf{z}_{i}^{\text {sol }}-\mathbf{x}\right),
$$

where $K_{\epsilon_{i}^{\text {sol }}}^{\perp}$ is a new kernel function obtained by convolving the orthogonal gradient of the Green kernel with the blob function. In the following, we will index all the parameters to distinguish the solenoidal part from the irrotational one.

We obtain a similar representation of the irrotational component using $q$ source particles and the Biot-Savart integral associated to the divergence map (equ.7):

$$
\mathbf{w}_{i r r}(\mathbf{x}) \approx \sum_{i=0}^{q} \gamma_{i}^{i r r} K * f_{\epsilon_{i}^{i r r}}\left(\mathbf{x}-\mathbf{z}_{i}^{i r r}\right)=\sum_{i=0}^{q} \gamma_{i}^{i r r} K_{\epsilon_{i}^{i r r}}\left(\mathbf{x}-\mathbf{z}_{i}^{i r r}\right) .
$$

As a result, we exhibit an approximation of the complete motion field as weighted sums of basis functions of two types. The basis functions, also called particles, are totally defined by their center location and respective spatial influence (varying with the parameter $\epsilon_{i}$ ). A strength 
coefficient $\gamma_{i}$ is associated to each particle. A Gaussian smoothing function allows to obtain an analytical expression of the associated smoothed kernel $K_{\epsilon}$ (equ.14). The final expressions of the motion field components are:

$$
\mathbf{w}_{\text {sol }}(\mathbf{x}) \approx \sum_{i=0}^{p} \gamma_{i}^{\text {sol }} \frac{\left(\mathbf{z}_{i}^{\text {sol }}-\mathbf{x}\right)^{\perp}}{2 \pi\left|\mathbf{x}-\mathbf{z}_{i}^{\text {sol }}\right|^{2}}\left(1-\exp \left(-\frac{\left|\mathbf{x}-\mathbf{z}_{i}^{\text {sol }}\right|^{2}}{\epsilon_{i}^{\text {sol }}}\right)\right),
$$

and

$$
\mathbf{w}_{i r r}(\mathbf{x}) \approx \sum_{i=0}^{q} \gamma_{i}^{i r r} \frac{\mathbf{x}-\mathbf{z}_{i}^{i r r}}{2 \pi\left|\mathbf{x}-\mathbf{z}_{i}^{i r r}\right|^{2}}\left(1-\exp \left(-\frac{\left|\mathbf{x}-\mathbf{z}_{i}^{i r r}\right|^{2}}{\epsilon_{i}^{i r r^{2}}}\right)\right) .
$$

Figure 2 depicts an example of a complex motion field representation obtained with 15 vortex particles and 5 source particles (that is to say, 80 parameters). In order to judge the complexity of the motion we also show the corresponding vorticity and divergence maps.

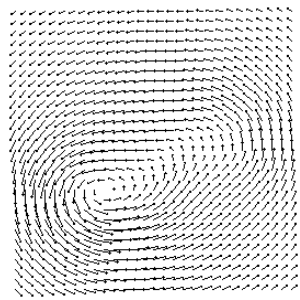

(a)

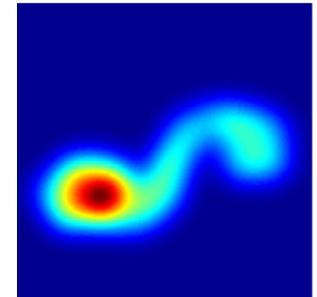

(b)

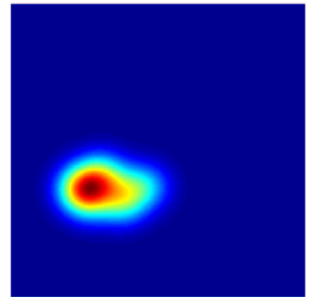

(c)

Figure 2: Fluid motion example. (a) Vector field expressed as a combination of vortex and source particles; (b) Corresponding vorticity map; (c) Corresponding divergence map.

In the next section, this representation is incorporated within a spatio-temporal variation model of the luminance function. The fluid motion recovery then becomes an estimation problem from the image sequence data.

\subsection{Integrated continuity equation as a brightness variation model}

Standard motion estimators rely on the hypothesis of the luminance conservation of a point along its trajectory $\left(\frac{d I}{d t}=0\right)$. Assuming this so-called optical flow constraint (OFC) is respected almost everywhere on the whole image domain $\Omega$ comes to find the minimizer of the following data cost function:

$$
\int_{\Omega} \phi\left[\nabla I(\mathbf{x}) \cdot \mathbf{w}(\mathbf{x})+\frac{\partial I(\mathbf{x})}{\partial t}\right] d \mathbf{x}
$$


The penalty function $\phi$ involved here is usually the $L_{2}$ norm but any robust function attenuating the effect of data that deviate significantly from the OFC-based data-model $[5,33]$ may be used instead. Without further specification on the motion field, the problem is ill-posed as we have to recover a vector function from a scalar constraint. In order to solve the problem a smoothness of the solution is generally imposed by considering an appropriate polynomial parameterization of the velocity field, or introducing various additional smoothing terms in the cost function $[13,25,42]$.

For image sequences showing evolving fluid phenomena, the usual brightness consistency assumption $\left(\frac{d I}{d t}=0\right)$ does not allow to model temporal distortions of luminance patterns caused by 3D matter transportation. For these sequences, it has been shown that a data model based on mass conservation (also known as the continuity equation) constitutes a better modeling $[2,4,13,38,39,43]$. The continuity equation links the variation of mass density, $\rho$, within an infinitesimal enclosing volume to the flux of matter through the volume boundary surface:

$$
\frac{\partial \rho}{\partial t}+\operatorname{div}(\rho \mathbf{v})=0
$$

Let us not that the vector $\mathbf{v}$ denotes a 3D velocity. Assuming strong hypothesis, it is possible to write a similar equation for a $2 \mathrm{D}$ representations of fluids:

$$
\frac{\partial I}{\partial t}+\operatorname{div}(I \mathbf{w})=0
$$

The hypothesis on which such a formulation relies are the following. First, the luminance function is assumed to be directly related to a passive quantity transported by the fluid ("density" images). Secondly, the continuity equation which holds in 3D is assumed to hold as well for the bidimensional motion field captured by the image sequence. This latter assumption has been theoretically established in the case of transmittance imaging by Fitzpatrick [20].

These two hypotheses cannot be easily justified for all types of images. Nevertheless, this continuity equation constitutes an interesting alternative to the brightness constancy equation. As a matter of fact, rewriting (23) as:

$$
\frac{d I}{d t}+I \operatorname{div} \mathbf{w}=0
$$

shows that such a constraint relates the effect of a divergent motion to a brightness change. By this way it is possible to modelize the effect of the apparent disappearance/appearance of matter caused by $3 \mathrm{D}$ motions which are not in the visualization plane. For compressible fluids, this data model relates directly temporal variations of the brightness function to the diverging nature 
of the velocity field. For a null divergence this data model reduces exactly to the brightness consistency equation.

For long range displacements (i.e. fast flows or long time latency between two images as in meteorology) an integrated form of this constraint can be obtained [13]:

$$
I(\mathbf{x}+\mathbf{w}(\mathbf{x}), t+1) \exp (\operatorname{div} \mathbf{w}(\mathbf{x}))-I(\mathbf{x}, t)=0 .
$$

According to this constraint, the displaced image at time $t+1$ is related to the image at time $t$ by a scale factor which depends on the motion divergence. This constraint comes to the standard displaced frame formulation of brightness consistency for a null divergence.

Finally, considering this constraint holds almost everywhere on the whole image plane and choosing the $L_{2}$ norm as penalty function leads to seek a motion field minimizing the following cost function:

$$
\mathcal{F}(I, \mathbf{w})=\int_{\Omega}[I(\mathbf{x}+\mathbf{w}(\mathbf{x}), t+1) \exp (\operatorname{div} \mathbf{w}(\mathbf{x}))-I(\mathbf{x}, t)]^{2} d \mathbf{x} .
$$

\subsection{General minimization problem}

Considering the previous cost function for an unknown motion field approximated through vortex and source particles representations comes down to solve the following minimization problem:

$$
\hat{\beta}=\arg \min _{\beta} \mathcal{F}(I, \mathbf{w}(\beta))
$$

with $\beta=\left(\left\{\mathbf{z}_{i}^{\text {sol }}, \gamma_{i}^{\text {sol }}, \epsilon_{i}^{\text {sol }}\right\}_{i=1: p},\left\{\mathbf{z}_{i}^{i r r}, \gamma_{i}^{i r r}, \epsilon_{i}^{i r r}\right\}_{i=1: q}\right)$. The variable $\mathbf{z}$ refers to the particle location, whereas $\gamma$ and $\epsilon$ represent respectively the strength coefficient and the influence domain.

One seeks therefore the minimizer of the cost function $\mathcal{F}$ in terms of particles locations, strength coefficients and influence domains. Due to the peculiar form of the data model, this minimization problem is highly non linear. In order to face up this difficult optimization problem we have chosen to rely on a non linear least square process embedded in a multiresolution framework and associated to a generalized conjugated gradient optimization known as FletcherReeves method.

We present more precisely in the next section how this difficult global optimization issue is handled. 


\section{Estimation}

The non linear cost function can be seen as a weighted displaced frame differences cost function. As most of the standard motion estimators based on such a non linear formulation, we will consider an incremental minimization framework to remove the non linearity of the displaced image brightness function $[1,5,6,35]$. This scheme consists in applying successive linearizations around previous estimates. This technique, similar to the Gauss-Newton non linear least squares, is in most cases embedded within a multiresolution framework. We will also rely on such a data representation.

\subsection{Incremental estimation scheme}

We first assume that a previous estimate of the set of unknown variables is available. All these unknowns interact with respect to our modelization to give a motion field $\widetilde{\mathbf{w}}$. Considering a linearization around $(\mathbf{x}+\widetilde{\mathbf{w}}, t+1)$ and dropping the time index of the intensity function for sake of clarity, we end up with the following functional, which should be minimized according to an unknown correction motion field denoted $\mathbf{h}$ :

$$
\mathcal{F}(I, \mathbf{h})=\int_{\Omega}\left[\exp (\operatorname{div} \tilde{\mathbf{w}})\left\{(\tilde{I} \boldsymbol{\nabla} \operatorname{div} \tilde{\mathbf{w}}+\nabla \tilde{I})^{T} \mathbf{h}+\tilde{I}\right\}-I\right]^{2} d \mathbf{x} .
$$

In this equation we have introduced a compact notation $\tilde{I}(\mathbf{x})$ for the backward registered image $I(\mathbf{x}+\widetilde{\mathbf{w}}, t+1)$. The correction field $\mathbf{h}$ is a combination of a solenoidal component $\mathbf{h}_{\text {sol }}$ and an irrotational component $\mathbf{h}_{\text {irr }}$ according to the Helmholtz decomposition. In the same way as $\widetilde{\mathbf{w}}$, the correction field $\mathbf{h}$ is parameterized on the basis of a set of vortex and source particles.

In practice, this kind of scheme is embedded into a pyramidal multiresolution data representation scheme [7]. Such a representation is obtained through low-pass filtering and subsampling. At a given level, the known motion estimate $\tilde{\mathbf{w}}$ is fixed to be the projected estimate obtained at the previous level. At the first level the initial field is a null field.

\subsection{Resulting minimization problem}

The incremental estimation scheme transforms the original non linear optimization problem (27) into a succession of simpler minimization problems with respect to some of the unknowns. As a matter of fact, considering the derivatives with respect to the different types of unknowns gives:

$$
\frac{\partial \mathcal{F}(\mathbf{h})}{\partial \gamma_{i}}=\int_{\Omega} \frac{\mathbf{r}_{i}}{\pi\left|\mathbf{r}_{i}\right|^{2}}\left(1-\exp \left(-\frac{\left|\mathbf{r}_{i}\right|^{2}}{\epsilon_{i}{ }^{2}}\right)\right) \mathbf{y}\left[\mathbf{y}^{T} \mathbf{h}\left(\gamma_{i}\right)+z\right] d \mathbf{x},
$$




$$
\begin{gathered}
\left.\frac{\partial \mathcal{F}(\mathbf{h})}{\partial \beta_{i}}\right|_{\beta_{i}=\frac{1}{\epsilon_{i}}}=\int_{\Omega} \frac{2 \gamma_{i}}{\pi \epsilon_{i}} \frac{\mathbf{r}_{i}}{\left|\mathbf{r}_{i}\right|^{2}} \exp \left(-\frac{\left|\mathbf{r}_{i}\right|^{2}}{\epsilon_{i}{ }^{2}}\right) \mathbf{y}\left[\mathbf{y}^{T} \mathbf{h}\left(\epsilon_{i}\right)+z\right] d \mathbf{x} \\
\nabla_{\mathbf{z}_{i}} \mathcal{F}(\mathbf{h})=\left(\begin{array}{c}
\frac{\partial \mathcal{F}(\mathbf{h})}{\partial x_{i}} \\
\frac{\partial \mathcal{F}(\mathbf{h})}{\partial y_{i}}
\end{array}\right),
\end{gathered}
$$

where:

$$
\begin{aligned}
\frac{\partial \mathcal{F}(\mathbf{h})}{\partial x_{i}}=\int_{\Omega} & -\frac{1}{\pi\left|\mathbf{r}_{i}(\mathbf{x})\right|^{4}} \frac{2}{\epsilon_{i}^{2}}\left|\mathbf{r}_{i}\right|^{2} r_{i}^{2}(x)+\left(\left|\mathbf{r}_{i}\right|^{2}+r_{i}^{2}(x)\right)\left(1-\exp \left(-\frac{\left|\mathbf{r}_{i}(\mathbf{x})\right|^{2}}{\epsilon_{i}^{2}}\right)\right) \\
& \mathbf{y}\left[\mathbf{y}^{T} \mathbf{h}\left(x_{i}\right)+z\right] d \mathbf{x} .
\end{aligned}
$$

In these expressions $\mathbf{r}_{i}, \mathbf{y}$ and $z$ are respectively vectors and function of $\mathbf{x}$ which are defined as:

$$
\left\{\begin{array}{l}
\mathbf{r}_{i}(\mathbf{x})=\left(r_{i}(x), r_{i}(y)\right)^{T}=\mathbf{x}-\mathbf{z}_{i}(\text { irr. part }) \text { or }\left(\mathbf{z}_{i}-\mathbf{x}\right)^{\perp}(\text { sol. part }) \\
\mathbf{y}(\mathbf{x})=\exp (\operatorname{div} \widetilde{\mathbf{w}}(\mathbf{x}))(\widetilde{I}(\mathbf{x}) \nabla \operatorname{div} \widetilde{\mathbf{w}}(\mathbf{x})+\nabla \widetilde{I}(\mathbf{x})) \\
z(\mathbf{x})=\exp (\operatorname{div} \widetilde{\mathbf{w}}(\mathbf{x})) \widetilde{I}(\mathbf{x})-I(\mathbf{x}, t)
\end{array}\right.
$$

Equations (29), (30) and (31) lead to three different kinds of systems. The first one is linear in terms of coefficient strengths, whereas the second one is non linear in terms of particles influence domains. Nevertheless, as no constrained minimization is required for both of them, a gradient descent process can be devised for this set of unknowns. For the third one an additional constraint must be added in order to keep the particles into the image plane. Such a constrained minimization problem, combined with the non linearity we have to face, would lead to a very tough minimization. Besides, if the initial particles locations are unknown, particles must be able to move far away from their initial positions. A gradient descent would not cope efficiently with such long moves.

As a consequence, we have splitted the resolution of these three kinds of unknowns. The two first types of variables (the strength coefficients and the influence domains of the particles) will be solved with a generalized conjugated gradient process described in section 5.3 while the third kind of unknowns (the particles locations) is kept fixed. The particles locations will be updated in turn through a mean shift process that is described in section 5.4. 


\subsection{Fletcher-Reeves optimization}

The Fletcher-Reeves method consists in a non linear extension of conjugate gradient algorithms. This method has shown its efficiency to solve general unconstrained minimization problems of large scale.

Given an iterate $\Theta_{k}=\left\{\gamma_{k}^{s o l}, \epsilon_{k}^{s o l}, \gamma_{k}^{i r r}, \epsilon_{k}^{i r r}\right\}$ and a descent direction $\mathbf{d}_{k}$, a line search (w.r.t. $\alpha_{k}$ ) is performed along $\mathbf{d}_{k}$ :

$$
f\left(\Theta_{k}+\alpha_{k} \mathbf{d}_{k}\right)=\min _{\alpha>0} f\left(\Theta_{k}+\alpha \mathbf{d}_{k}\right),
$$

and leads to $\Theta_{k+1}=\Theta_{k}+\alpha_{k} \mathbf{d}_{k}$. The descent direction $\mathbf{d}_{k+1}$ is generated with the recursive form:

$$
\mathbf{d}_{k+1}=-\nabla \mathcal{F}\left(\Theta_{k+1}\right)+\mu_{k} \mathbf{d}_{k}
$$

with:

$$
\mu_{k}= \begin{cases}0 & \text { for } k=1, \\ \left(\frac{\left\|\nabla \mathcal{F}\left(\Theta_{k+1}\right)\right\|_{2}}{\left\|\boldsymbol{\nabla} \mathcal{F}\left(\Theta_{k}\right)\right\|_{2}}\right)^{2} & \text { for } k \geq 2,\end{cases}
$$

where $\mu_{k}$ is a scalar, corresponding to the Fletcher-Reeves variant of the non linear conjugate gradient algorithm. Various forms of this coefficient exist (see for example [23]) leading to different versions of the algorithm. Different implementation schemes may be chosen, based on exact or inexact line searches, or on the possibility to restart the iteration (35) every $n$ steps by setting $\mu_{k}$ to zero (i.e. imposing a steepest descent strategy). We rely here on a method based on an exact line search, without restarting. The global convergence of such a method is studied in [23].

Let us note that for the linear part of our system, the method comes to the standard conjugate gradient method. To start the optimization, we consider, as said before, that initial particle locations are known. The initial strength coefficients are set to zero and we choose an adaptive ad hoc strategy for the initialization of the influence domains. We fix their values to the value of the distance to the nearest particle. At convergence, we obtain a representation of the unknown correction field for fixed particle locations. Let us now describe how we propose to adjust these locations.

\subsection{Adjustment of particles location}

The estimation method we have described in the previous section requires to fix particles locations on the image domain for the solenoidal and irrotational components. We now propose 
a way to move each particle according to a characteristic surface defined from the image data. The method we propose is based on the mean shift procedure.

\subsubsection{Definition of the error function}

Considering that estimates of the strength coefficients and influence domains are available for both irrotational and solenoidal components, we consider two different error surfaces. For each component, the surface corresponds to the registration discrepancy, considering the other orthogonal component fixed. For the solenoidal component the error surface is thus defined at each point of the image domain as:

$$
\mathcal{D}_{k}^{s o l}(\mathbf{x})=I_{t+1}\left(\mathbf{x}+\widetilde{\mathbf{w}}(\mathbf{x})+\widetilde{\mathbf{h}}_{k}^{i r r}(\mathbf{x})\right)-I_{t}(\mathbf{x}),
$$

where $\widetilde{\mathbf{h}}_{k}^{i r r}$ is an estimate of the irrotational increment at step $k$. This error surface gathers the main reconstruction errors due to the solenoidal component. Similarly the error surface corresponding to the irrotational component is defined as:

$$
\mathcal{D}_{k}^{i r r}(\mathbf{x})=I_{t+1}\left(\mathbf{x}+\widetilde{\mathbf{w}}(\mathbf{x})+\widetilde{\mathbf{h}}_{k}^{s o l}(\mathbf{x})\right)-I_{t}(\mathbf{x})
$$

\subsubsection{Extension to a characteristic surface}

The quality of the modelization depends on the accuracy of the discrete approximation of the divergence and curl maps. To achieve the best approximation with a limited number of particles, many particles should be used to describe areas with strong divergence or vorticity and only few of them for the rest of the image. The surface error, as defined by (37) or (38), can help to guide a particle toward a new location in accordance with its nature (vortex or source). However a given particle can be guided toward an inappropriate location when the error surface reveals errors associated to the two irrotational and solenoidal components and not only to one of them. In order to overcome this problem, we have an additional term for each error surface. This term takes into account the amount of estimated vorticity or divergence. Particles are then encouraged to go toward locations of high error magnitude associated to high concentration of vorticity or divergence. These two kinds of surfaces are normalized and combined to create two global error surfaces for the solenoidal and the irrotational parts:

$$
\mathcal{S}_{k}^{s o l}(\mathbf{x})=\left(\mathcal{D}_{k}^{s o l}(\mathbf{x})\right)^{2}+\left(\operatorname{curl} \widetilde{\mathbf{h}}_{k}(\mathbf{x})\right)^{2},
$$

and

$$
\mathcal{S}_{k}^{i r r}(\mathbf{x})=\left(\mathcal{D}_{k}^{i r r}(\mathbf{x})\right)^{2}+\left(\operatorname{div} \widetilde{\mathbf{h}}_{k}(\mathbf{x})\right)^{2}
$$


Finally, in order to restrict the displacements of the different particles to localized areas, we combine these functions with an a priori on the particles location.

\subsubsection{A priori probability distribution for particles locations}

Considering $\mathbf{z}_{k}^{(i)}$ the vector denoting the location of particle $i$ at step $k$, we propose to fix a distribution of $\mathbf{z}_{k+1}^{(i)}$, knowing $\mathbf{z}_{k}$, where $\mathbf{z}_{k}$ represents the set of the $n$ positions $\left(\mathbf{z}_{k}^{(1)}, \ldots, \mathbf{z}_{k}^{(n)}\right)$ (where $n$ equals $p$ or $q$ ) at step $k$. We will assume that the associated probability density function is Gaussian, defined as follows:

$$
\mathbf{z}_{k+1}^{(i)} \mid \mathbf{z}_{k} \sim \mathcal{N}\left(\mathbf{z}_{k}^{(i)}, \sigma_{k}^{(i)}\right)
$$

The standard deviation $\sigma_{k}^{(i)}$ is set to the distance between $\mathbf{z}_{k}^{(i)}$ and the closest center among $\left\{\mathbf{z}_{k}^{(j)}\right\}_{j=1, . ., n, j \neq i}$. Let us note that this distribution takes into account the previous location of the particle through a Gaussian prior of mean $\mathbf{z}_{k}^{(i)}$ but also the dependency between $\mathbf{z}_{k+1}^{(i)}$ and all the other particles through $\sigma_{k}^{(i)}$.

\subsubsection{Conditional version of the probability distribution}

Combining the a priori distribution $p_{\mathbf{z}_{k+1}^{(i)} \mid \mathbf{z}_{k}}$ defined above with the surface described before, denoted $\mathcal{S}_{k}$ in the general case and characterized by (39) or (40), we can define a conditional probability distribution function of a particle $\mathbf{z}_{k+1}^{(i)}$ given the others:

$$
p_{\mathbf{z}_{k+1}^{(i)} \mid \mathbf{z}_{k}, \mathcal{S}_{k}}(\mathbf{x}) \propto \mathcal{S}_{k}(\mathbf{x}) \cdot p_{\mathbf{z}_{k+1}^{(i)} \mid \mathbf{z}_{k}}(\mathbf{x}) \hat{=} f^{(i)}(\mathbf{x}) .
$$

This pdf balances an a priori for the location of one given particle (whose role is to confine the particle to stay in a certain area between two iterates) and the information brought by the characteristic surface (associated to all the particles locations) in the neighborhood of this position. In order to adjust optimally the particles positions we propose to shift each center $\mathbf{z}_{k}^{(i)}$ toward the nearest mode of the surface $f^{(i)}$. We present in the next section the methodology used to shift each center.

\subsubsection{Shifting the particles toward the pdf modes}

Mean shift procedure The mean shift procedure was first presented by [22], extended by [8] and popularized in image processing by [11]. It describes how to shift iteratively a point toward the nearest mode of a probability distribution function. This procedure relies on a non parametric estimate of the gradient of the underlying density function. 
The value of a density function can be estimated at a point using the sample observations that fall within a small region around this point. In fact, given a sample $\mathbf{x}_{1}, \ldots, \mathbf{x}_{n}$, a kernel density estimate can be used to represent the density as follows:

$$
\hat{f}_{\mathcal{K}}(\mathbf{x})=\frac{1}{n h^{d}} \sum_{i=1}^{n} \mathcal{K}\left(\frac{\mathbf{x}-\mathbf{x}_{i}}{h}\right) .
$$

A kernel function $\mathcal{K}$ is an $\mathbb{R}^{d} \rightarrow \mathbb{R}$ application which is generally symmetric, bounded and such that $\int_{-\infty}^{+\infty} \mathcal{K}(\mathbf{x}) d \mathbf{x}=1$. The kernels considered here are radial functions such that $\mathcal{K}(\mathbf{x})=$ $k\left(\|\mathbf{x}\|^{2}\right)$.

From expression (43) the gradient of the estimated density reads:

$$
\begin{aligned}
\hat{\boldsymbol{\nabla}} f_{\mathcal{K}}(\mathbf{x}) & =\boldsymbol{\nabla} \hat{f}_{\mathcal{K}}(\mathbf{x})=\frac{1}{n h^{d}} \sum_{i=1}^{n} \boldsymbol{\nabla} \mathcal{K}\left(\frac{\mathbf{x}-\mathbf{x}_{i}}{h}\right) \\
& =\frac{2}{n h^{d+2}} \sum_{i=1}^{n}\left(\mathbf{x}-\mathbf{x}_{i}\right) k^{\prime}\left(\left\|\frac{\mathbf{x}-\mathbf{x}_{i}}{h}\right\|^{2}\right) \\
& =\frac{2}{n h^{d+2}}\left[\sum_{i=1}^{n} g\left(\left\|\frac{\mathbf{x}-\mathbf{x}_{i}}{h}\right\|^{2}\right)\right]\left[\frac{\sum_{i=1}^{n} \mathbf{x}_{i} g\left(\left\|\frac{\mathbf{x}-\mathbf{x}_{i}}{h}\right\|^{2}\right)}{\sum_{i=1}^{n} g\left(\left\|\frac{\mathbf{x}-\mathbf{x}_{i}}{h}\right\|^{2}\right)}-\mathbf{x}\right]
\end{aligned}
$$

where $g$ is a function such that $g(x)=-k^{\prime}(x)$ and $\mathcal{G}$ is a kernel defined as $\mathcal{G}(\mathbf{x})=c g\left(\|\mathbf{x}\|^{2}\right)$, with $c$ normalization constant. We can recognize in the first bracket the density at $\mathbf{x}$ estimated with the kernel $\mathcal{G}$ :

$$
\hat{f}_{\mathcal{G}}(\mathbf{x})=\frac{c}{n h^{d}} \sum_{i=1}^{n} g\left(\left\|\frac{\mathbf{x}-\mathbf{x}_{i}}{h}\right\|^{2}\right)
$$

and the so-called mean shift vector in the second bracket:

$$
\mathbf{m}_{\mathcal{G}, h}(\mathbf{x})=\frac{\sum_{i=1}^{n} \mathbf{x}_{i} g\left(\left\|\frac{\mathbf{x}-\mathbf{x}_{i}}{h}\right\|^{2}\right)}{\sum_{i=1}^{n} g\left(\left\|\frac{\mathbf{x}-\mathbf{x}_{i}}{h}\right\|^{2}\right)}-\mathbf{x} .
$$

It follows that:

$$
\mathbf{m}_{\mathcal{G}, h}(\mathbf{x}) \propto \frac{\hat{\nabla} f_{\mathcal{K}}(\mathbf{x})}{\hat{f}_{\mathcal{G}}(\mathbf{x})}
$$


The mean shift vector (44), which is the difference between the weighted mean evaluated with the kernel $\mathcal{G}$ and the center of the kernel, can then be seen as a normalized estimate of the pdf gradient. Because this mean shift vector gives at every point the direction of the maximum increase of the density function, an iterative process naturally arises. It consists in moving iteratively the kernel center $\mathbf{x}$ following $\mathbf{m}_{h, \mathcal{G}}(\mathbf{x})$ until a stationary point (i.e. zero gradient) of the underlying density is found. The process is convergent if the kernel $\mathcal{K}$ has a convex and decreasing profile $k$ (see [11]).

Kernel density estimate of the conditional distribution To apply the mean shift procedure to the surface $f^{(i)}$ we first need to represent it as a kernel density estimate. Let $\mathbf{y}$ represent a pixel coordinate in the image domain $\Omega$. The value $f^{(i)}(\mathbf{y})$ can be computed for each $\mathbf{y}$ using formula (42) where $\mathcal{S}_{k}(\mathbf{y})$ is given by (39) or (40). A non parametric estimate of the conditional distribution may then be obtained, expressed as a weighted average of the observations that fall within the window of $\mathcal{K}$ :

$$
\hat{p}_{\mathbf{z}_{k+1}^{(i)} \mid \mathbf{z}_{k}, \mathcal{S}_{k}}(\mathbf{x})=\frac{\sum_{\mathbf{y} \in \Omega} f^{(i)}(\mathbf{y}) \mathcal{K}\left(\frac{\mathbf{x}-\mathbf{y}}{h}\right)}{\sum_{\mathbf{y} \in \Omega} \mathcal{K}\left(\frac{\mathbf{x}-\mathbf{y}}{h}\right)} .
$$

Note here that the denominator does not depend on $\mathbf{x}$, since the pixels $\mathbf{s}$ are disposed regularly on the image and we assume that $h$ is chosen independently from $\mathbf{x}$. We can then see (45) as a density estimate of the form (43), where each possible value $\mathbf{y}$ is weighted by $w(\mathbf{y})=$ $\frac{f^{(i)}(\mathbf{y})}{\sum_{\mathbf{y} \in \Omega} \mathcal{K}\left(\frac{\mathbf{x}-\mathbf{y}}{h}\right)}$. This remark is important since it will allow us to apply a modified expression of the mean shift procedure [12], as described in the next paragraph.

Application of the mean shift procedure These weights $w(\mathbf{y})$ can be included in the expression of the mean shift vector (44) as follows:

$$
\mathbf{m}_{\mathcal{G}, h}(\mathbf{x})=\frac{\sum_{\mathbf{y} \in \Omega} \mathbf{y} w(\mathbf{y}) g\left(\left\|\frac{\mathbf{x}-\mathbf{y}}{h}\right\|^{2}\right)}{\sum_{\mathbf{y} \in \Omega} w(\mathbf{y}) g\left(\left\|\frac{\mathbf{x}-\mathbf{y}}{h}\right\|^{2}\right)}-\mathbf{x} .
$$

The iterative process to a stationary point of the underlying density estimate remains the same, using this modified expression for the mean shift vector. Note that the convergence of the mean 
shift process can also be demonstrated when such a positive weight is associated to each data [12].

The last step before applying the mean shift procedure is the choice of the kernel $\mathcal{K}$. We choose the Epanechnikov kernel, given by $\mathcal{K}(x)=\frac{3}{4 \sqrt{5}}\left(1-\frac{x^{2}}{5}\right)$ for $|x|<\sqrt{5}$. It is known to be optimal under some conditions, minimizing the asymptotic mean integrated square error between the density and its estimate. Moreover, it is practical and interesting since the derivative $g$ of its profile is simply uniform (box function kernel), which makes the evaluation of the mean shift vector (46) simple and fast. Besides, the choice of the window size is crucial. Different choices can be made. In this work, we have settled adaptive window sizes. They are fixed to the distance of the nearest particle and will be adapted after all centers have moved. Such a choice makes sense in our case. As a matter of fact, for distant particles only a rough and smooth estimate of the pdf function is needed whereas for close particles an accurate estimate of the density is at the opposite required to approximate at best the vorticity and divergence maps.

We finally apply the mean shift procedure to the $p+q$ centers of the basis functions (vortex or source particles) involved in our motion field modelization. Through this process, each particle's center $\mathbf{z}_{k}^{(i)}$ is shifted toward the nearest mode of the conditional estimated density $\hat{p}_{\mathbf{z}_{k+1}^{(i)} \mid \mathbf{z}_{k}, \mathcal{S}_{k}}$.

\subsection{Overall estimation scheme}

For each level of the multiresolution image pyramid, the overall estimation scheme consists in an alternate updating of the different unknowns. It is composed of the following two steps, repeated in turn until convergence:

1. For a given set of particles at fixed locations $\mathbf{z}_{k}=\left(\mathbf{z}_{k}^{(1)}, \ldots, \mathbf{z}_{k}^{(n)}\right)$, the strength coefficients and the influence domains attached to the particles are estimated through the generalized conjugate gradient optimization described in section 5.3. It gives an estimate of the incremental field $\widetilde{\mathbf{h}}_{k}$.

2. Using the information given by $\mathbf{z}_{k}$ and $\widetilde{\mathbf{h}}_{k}$, the vortex and source particles locations are shifted toward the nearest local mode of the conditional pdf (42). These shifts are realized applying the mean shift procedure as described in section 5.4, and lead to a set of new positions $\mathbf{z}_{k+1}=\left(\mathbf{z}_{k+1}^{(1)}, \ldots, \mathbf{z}_{k+1}^{(n)}\right)$. 
The whole process is stopped when the divergence and vorticity reach a certain stability. This criterion is expressed as:

$$
\left(\frac{\left\|\operatorname{div} \widetilde{\mathbf{h}}_{k+1}-\operatorname{div} \widetilde{\mathbf{h}}_{k}\right\|_{2}}{\left\|\operatorname{div} \widetilde{\mathbf{h}}_{k}\right\|_{2}}\right)^{2}+\left(\frac{\left\|\operatorname{curl} \widetilde{\mathbf{h}}_{k+1}-\operatorname{curl} \widetilde{\mathbf{h}}_{k}\right\|_{2}}{\left\|\operatorname{curl} \widetilde{\mathbf{h}}_{k}\right\|_{2}}\right)^{2}
$$

\section{Experimental results}

We have carried out some experiments on synthetic data and on real world images to demonstrate the relevance of the proposed method .

\subsection{Synthetic examples}

Synthetic field created from vortex and source particles We first present a synthetic example composed of a rotation, a divergent motion (source), and a combination of a vortex and a sink. The synthetic field is presented in figure 3 with the corresponding vorticity and divergence maps. The example has been created with 2 vortex particles and 2 source particles.

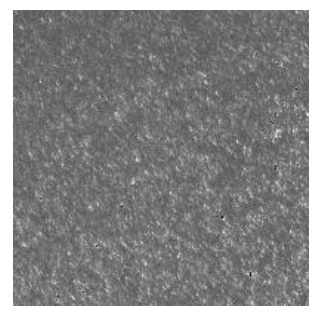

(a)

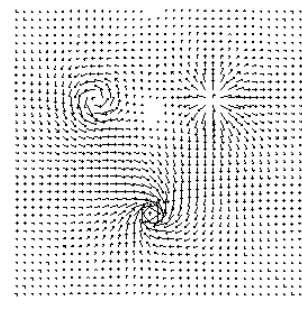

(b)

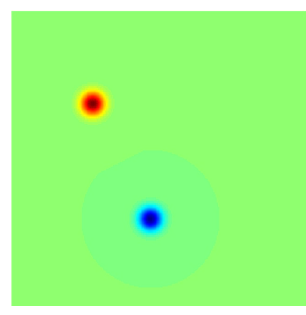

(c)

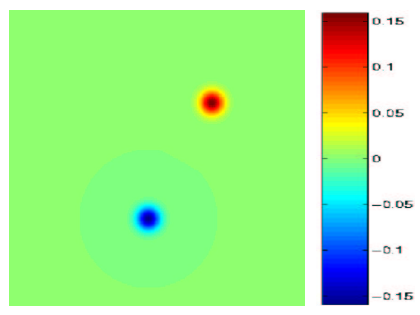

(d)

Figure 3: Synthetic example with ground truth. (a) PIV image; (b) Parametric motion field; (c) Vorticity map (mean absolute vorticity $=1.510^{-3}$ ); (d) Divergence map (mean absolute divergence $=1.510^{-3}$ ).

In order to create a test sequence with ground truth the synthetic vector field has been applied to an image of a fluid flow seeded with particles. That kind of images is currently used to estimate flow velocity in experimental fluid mechanics. The corresponding correlation based motion estimation techniques are usually referred as PIV techniques (Particle Image Velocimetry). We present results obtained on the pair of images composed of the original particle image and the backward registered image according to the considered motion field. We first show on figure 4 the result obtained when the true positions of the 4 particles are known. The strength of each particle has been initialized to zero and the influence to the distance to the nearest particle. 
We can observe that the estimation method allows to estimate very accurately the actual motion field and the vorticity and divergence carried by each particle. The mean absolute values of vorticity and divergence (see the legend figure 4) can be compared to the true ones (see the legend figure 3).

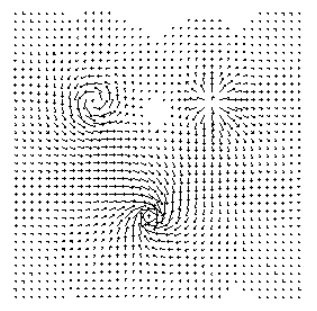

(a)

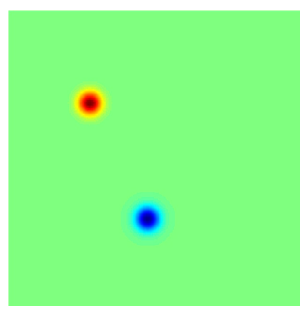

(b)

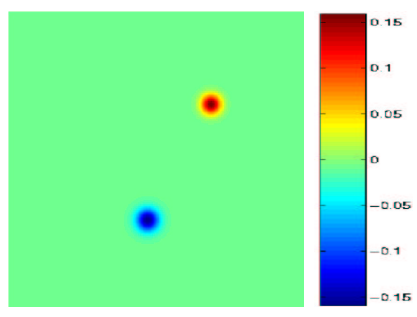

(c)

Figure 4: Result with known initial positions of the particles. (a) Estimated motion; (b) Estimated vorticity (mean absolute value $=1.410^{-3}$, mean absolute error $=1.410^{-4}$ ); (c) Estimated divergence $\left(\right.$ mean absolute value $=1.310^{-3}$, mean absolute error $=2.710^{-4}$ ).

We show in figure 5 the estimation result when the algorithm is initialized with several perturbed positions in the neighborhood of the true centers of the 4 synthetic particles. Even if 4 particles are necessary to represent the motion field, we wanted here to test also the robustness to an over-particles representation (12 particles here). We can observe on figure 5 that the mean shift procedure coupled with the estimation of the strength and influence parameters allows to converge iteratively toward the actual positions. All the supplementary particles converge to the same location. On figure 6 we picture the evolution of the divergence and vorticity maps during the whole estimation process. In this case five successive alternate iterations have been needed to reach convergence. This simple experiment demonstrates the robustness of the particles location adaptation with respect to an over-representation and also to an inaccurate initialization, even if the mean absolute errors for the estimation of the divergence and the vorticity are a bit higher for this perturbed case (see the legend of figure 6). 


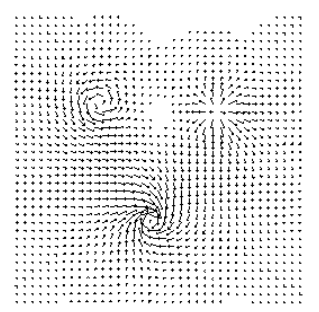

(a)

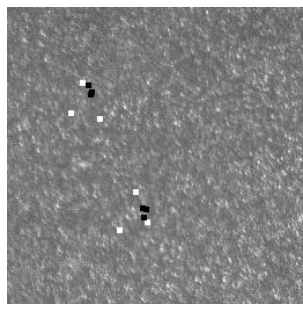

(b)

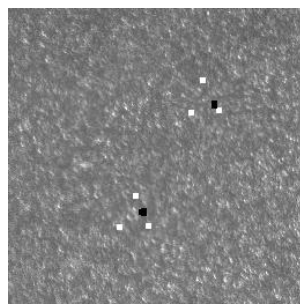

(d)

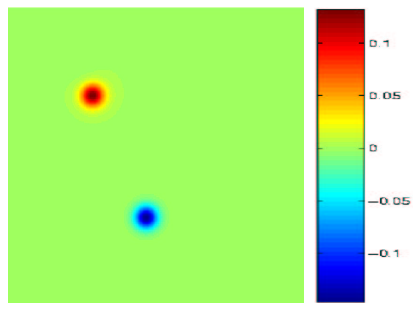

(c)

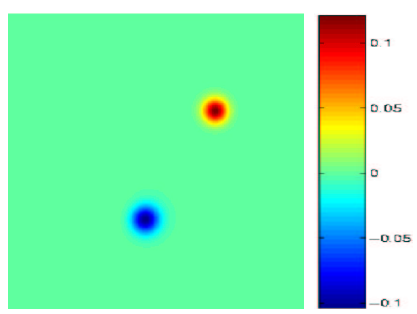

(e)

Figure 5: Result with perturbed initial positions of the particles. (a) Estimated motion field; (b) The initial positions of the vortex particles are indicated in white, positions at the end of the algorithm in black; (c) Estimated vorticity (mean absolute value $=1.410^{-3}$, mean absolute error $=3.410^{-4}$ ); (d) Initial and final positions of the source particles; (e) Estimated divergence $\left(\right.$ mean absolute value $=1.310^{-3}$, mean absolute error $=5.310^{-4}$ ). 


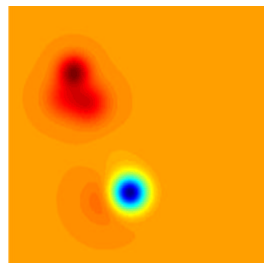

$k=0$

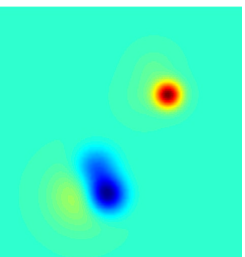

$k=0$

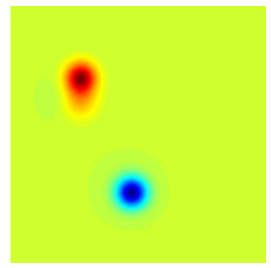

$k=1$

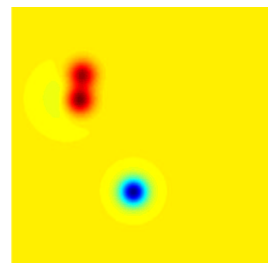

$k=2$

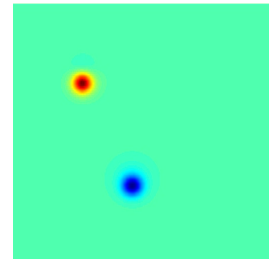

Evolution of vorticity maps

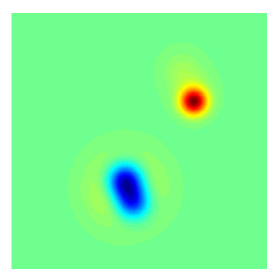

$k=1$

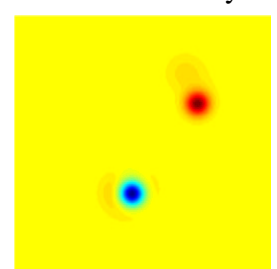

$k=2$ $k=3$

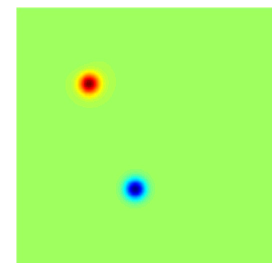

$k=4$

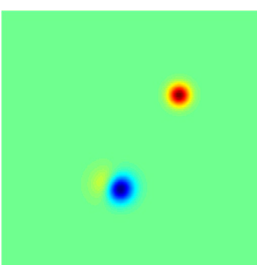

$k=3$

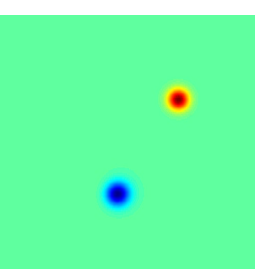

$k=4$

Evolution of divergence maps

Figure 6: Evolution of the estimation of the vorticity and divergence maps along the iterations of the two steps scheme (estimation of the vorticity or divergence carried by each particle / adjustment of the centers by the mean shift procedure).

Synthetic field created from a real fluid flow We present a second synthetic example created from a complex motion field describing the $2 \mathrm{D}$ evolution of a turbulent wake behind a circular cylinder. The field has been obtained by simulation of the Navier-Stokes equation with a DNS method (Direct Numerical Simulation) [27]. A synthetic pair is created from one initial image of particles (see figure 7 (a)). The known displacement and the associated vorticity map are represented figure $7(\mathrm{~b}, \mathrm{c})$. To compare the accuracy of the different results, we compute the mean absolute vorticity over the image and the mean absolute error between the true scalar vorticity and the estimated one. We first show in figure 8 the result obtained by a dense estimation method dedicated to fluid flows [13]. The resulting motion field is presented in figure 8 (a), with the corresponding vorticity map figure $8(\mathrm{~b})$. We can remark that the motion field provided by the dense estimation method shows a profile which is very close to the ground truth, but local estimation errors appear on the recovered vorticity map. 


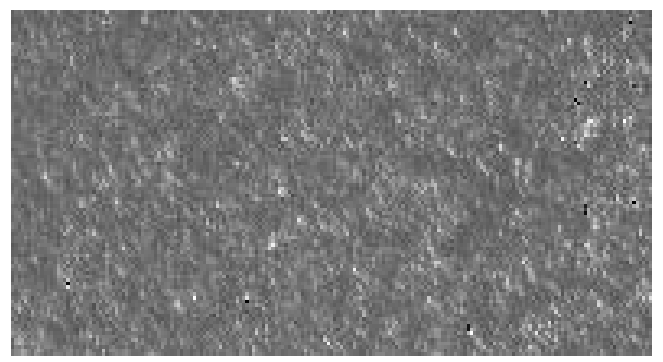

(a)

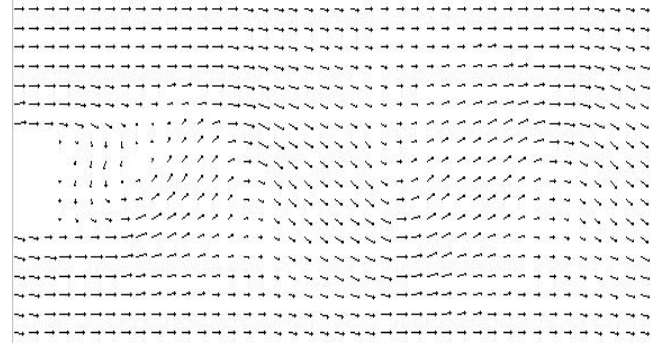

(b)

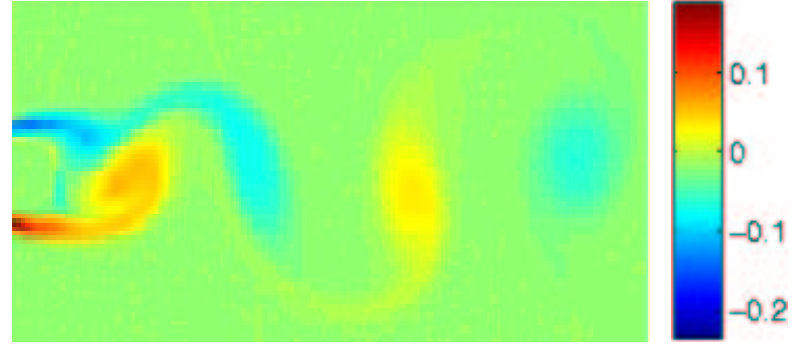

(c)

Figure 7: Synthetic example obtained by DNS ( Direct Numerical Simulation). (a) PIV image; (b) Synthetic motion field obtained by simulation of the Navier-Stokes equation; (c) Vorticity map (mean absolute vorticity $=0.0117$ ).

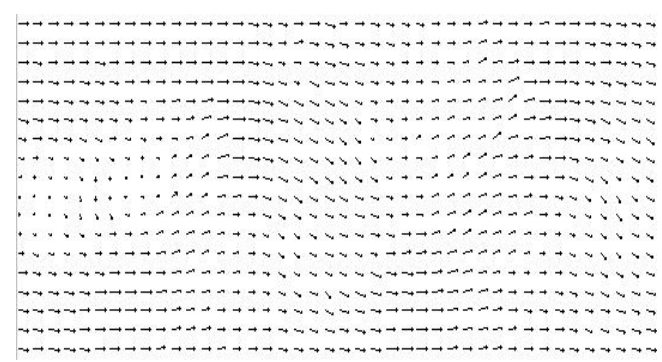

(a)

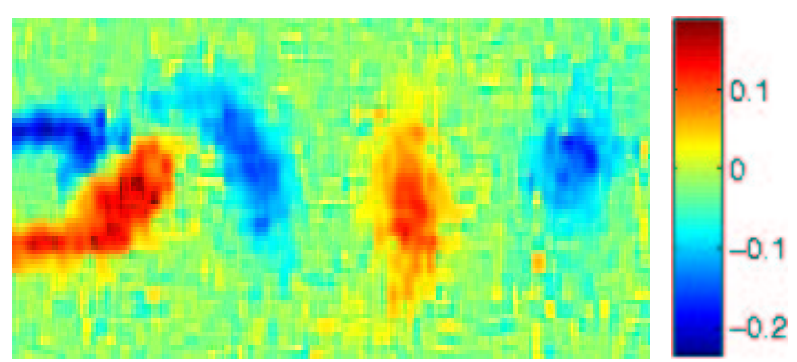

(b)

Figure 8: Result obtained by a dedicated dense method (see [13]) (a) Estimated vector field; (b) Associated vorticity map (mean absolute vorticity $=0.0172$, mean absolute error $=0.012$ ).

Figure 9 shows the result obtained by our parametric method with a initial uniform grid of $30 * 50$ vortex particles. Note that the positions are kept fixed on the grid during the estimation process, since the grid is dense and the vorticity regions are already recovered with particles. The global transportation harmonic field has been first extracted (see figure 9(a)). This component has been estimated with a standard dense motion estimator associated to a strong regularization 
coefficient and removed from the image sequence. The values of the mean absolute vorticity and the mean absolute error (see the legend figure 9) can be compared to the dense method result (see the legend figure 8 ). The dense method seems to be more noisy and subject to small local errors, this can explain the fact that the corresponding mean absolute error is higher.

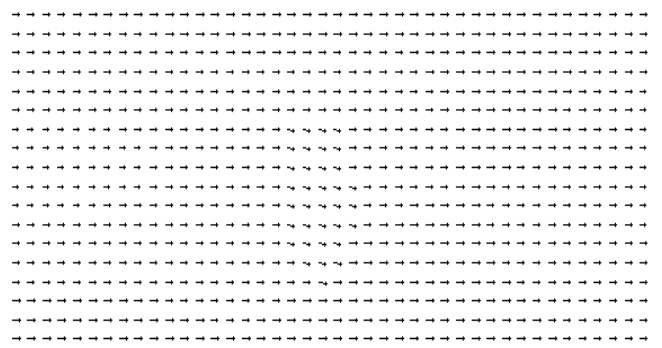

(a)

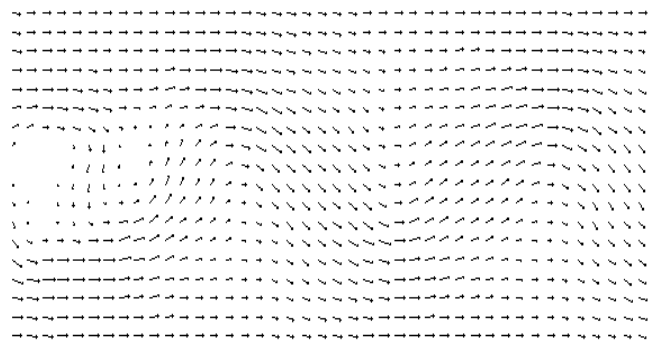

(c)

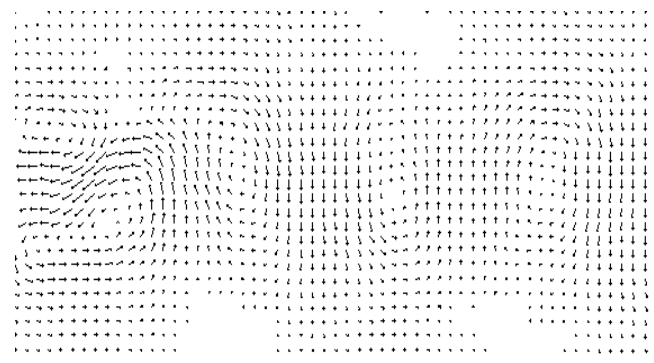

(b)

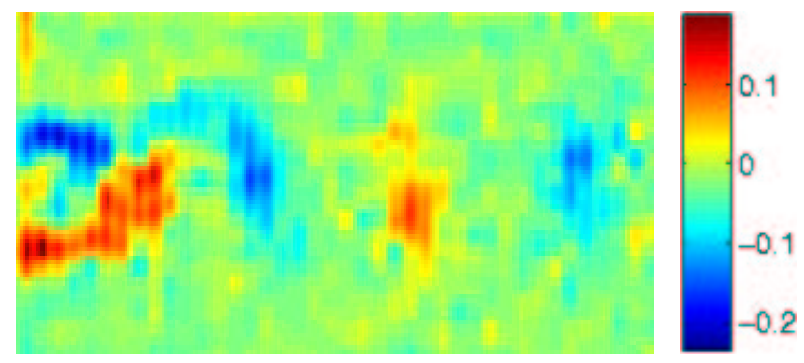

(d)

Figure 9: Result with a high number of vortex particles (a) Estimated transportation component; (b) Estimated solenoidal vector field; (c) Complete estimated vector field, sum of the transportation and solenoidal components; (d) Vorticity map (mean absolute vorticity = 0.0159 , mean absolute error $=0.0106)$.

To illustrate the results that can be obtained considering a much lower number of particles, we show an experiment carried out with a minimal number of vortex particles. Only 6 particles have been used to estimate the motion field presented figure $10(\mathrm{~b})$, with initial positions fixed on a central line on the image. After adding the transportation component, the corresponding motion field is shown figure 10 (c). The vorticity distribution associated to the 6 vortex particles is represented figure $10(d)$. We can remark that even if the estimated vorticity map consists in a crude representation of the real vorticity map (see figure 7 (c)), the corresponding motion field is close to the real one. The solution is less subject to local estimation errors, leading to a smaller mean absolute error for the vorticity (see the legend of figure 10). This result highlights the fact that a coherent motion field can be recovered by our method with only a very few number 
of particles. This low dimensional representation of the motion field enables a meaningful and direct interpretation of the organization and location of vortices in the flow. It can also be pointed out that such a low dimensional representation can be estimated with a computational cost much lower than the dense method. This is no longer the case for a representation with a very large number of particles, where the computational cost of the proposed method is higher.

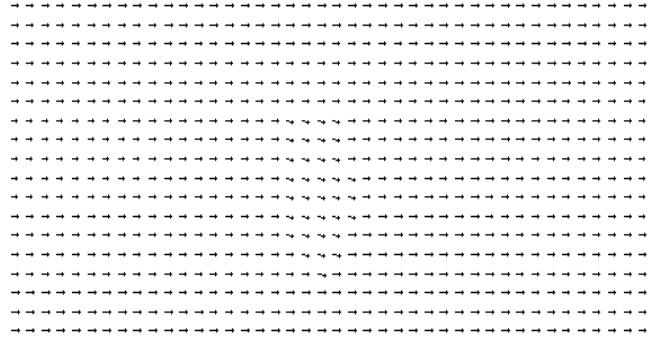

(a)

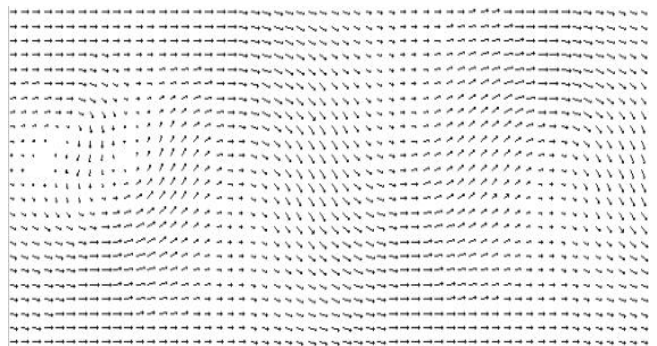

(c)

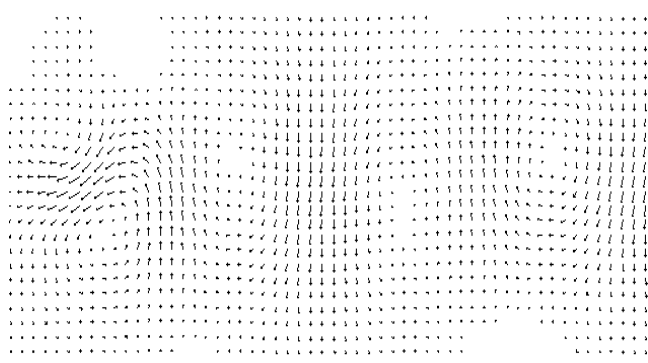

(b)

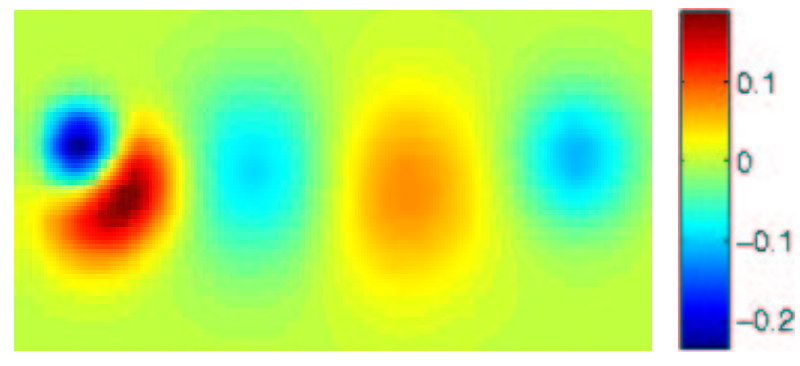

(d)

Figure 10: Result with a minimal number of vortex particles (a) Estimated transportation component; (b) Estimated solenoidal vector field; (c) Complete estimated vector field, sum of the transportation and solenoidal components; (d) Vorticity map (mean absolute vorticity $=0.011$, mean absolute error $=0.0091$ ).

Synthetic images of a 2D turbulent flow This example deals with synthetic images of particles transported by a complex $2 \mathrm{D}$ turbulent flow with many interacting vortices. The true displacement and the corresponding vorticity map are shown figure $11(a, b)$. This flow has also been simulated through a DNS technique [27]. The motion is purely rotational and there is here no transportation component. We show in figure $11(c, d)$ the result obtained by the dense estimation method [13]. The solution is compared to our parametric estimation result, with a grid of $20 * 20$ particles (see figure $11(e, f)$ ). A global quantitative comparison can be done looking at the values of mean absolute vorticity and mean absolute error (see the legend of figure 11). The mean absolute error is lower for the dense estimator, while our method provides 
a solution with a mean absolute vorticity closer to the truth. We have performed a finer comparison, in order to highlight more precisely the differences between these two different estimators. We plot on figure 12 the contour lines associated to a given value of absolute vorticity, for the reference map and the two different estimation methods respectively. These contours stress the fact that the dense method is more subject to small local errors, while our parametric estimator allows us to recover large scale vorticity structures and tends to smooth the fine scale structures. These small structures are present in the solution obtained from the dense estimator, nevertheless they are very difficult to separate from high frequencies generated by estimation errors (see figure 12). If one aims at characterizing large and medium vortical structures of the flow, the low dimensional estimator appears to be much more convenient to use. In order to confirm further this assertion, we have plotted on figure 13 two vorticity profiles for two given lines in the image. On the graphics shown in this figure we have superimposed the vorticity profile obtained by the dense optical flow method and the parametric motion estimator to the actual vorticity profile. We can see that the profiles corresponding to the dense estimation are very close to the solution but present fine scale estimation errors, while the parametric solution profiles present indeed a smoothed version of the solution. The parametric estimation does not allow to capture all the details of small scales of vorticity, but enables to represent quite accurately the large scale components of the flow. This is a precious property for analyzing or characterizing the large eddies of the flow. 


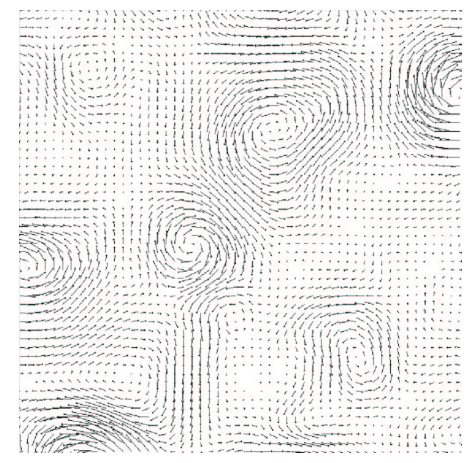

(a)

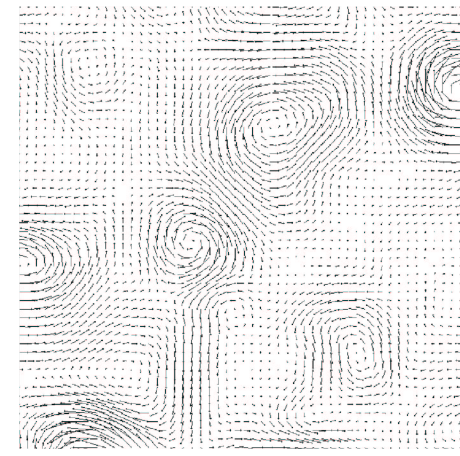

(c)

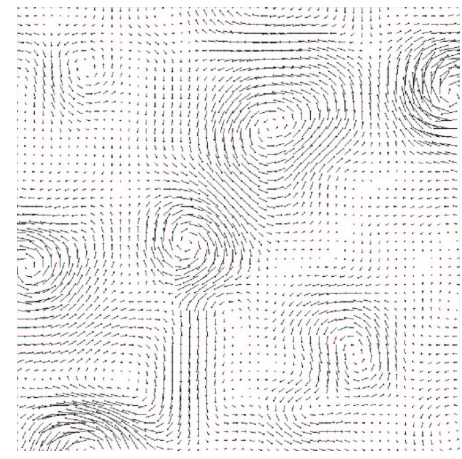

(e)

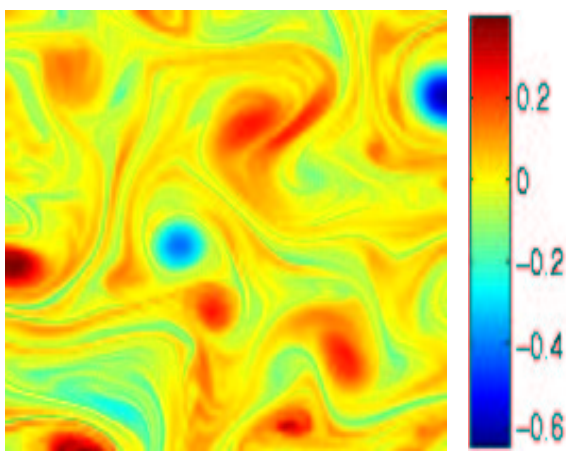

(b)

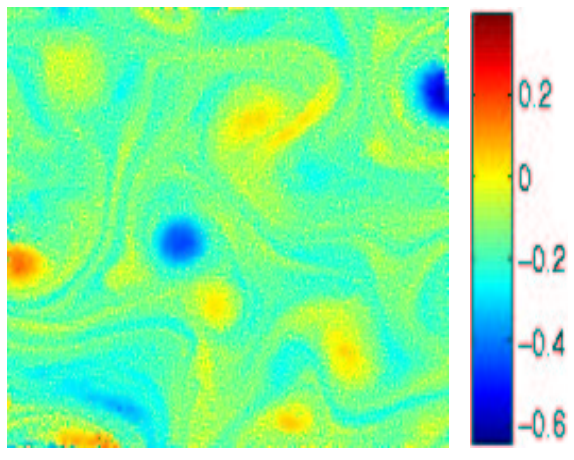

(d)

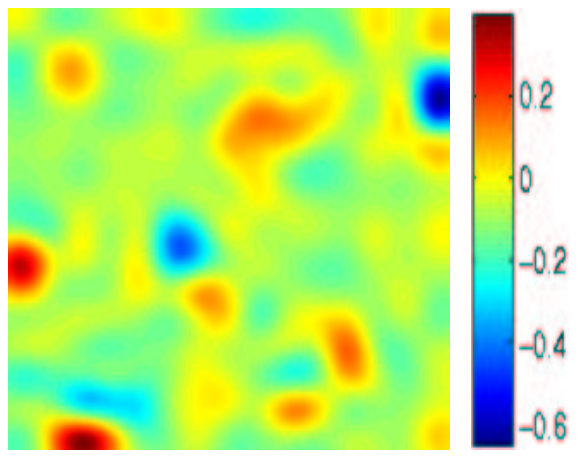

(f)

Figure 11: $2 D$ turbulent flow. (a) True motion field; (b) Vorticity map (mean absolute vorticity $=0.0639$ ); (c) Motion field estimated by a dedicated dense method [13]; (d) Associated vorticity map (mean absolute vorticity $=0.0663$, mean absolute error $=0.0286$ ); (e) Motion field estimated by the proposed technique; ( $\mathrm{f}$ ) Associated vorticity map (mean absolute vorticity $=0.062$, mean absolute error $=0.0363$ ). 


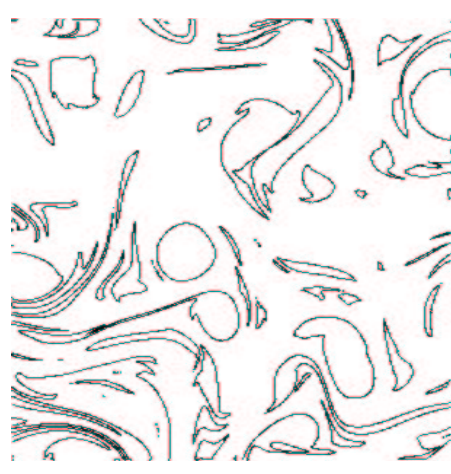

(a)

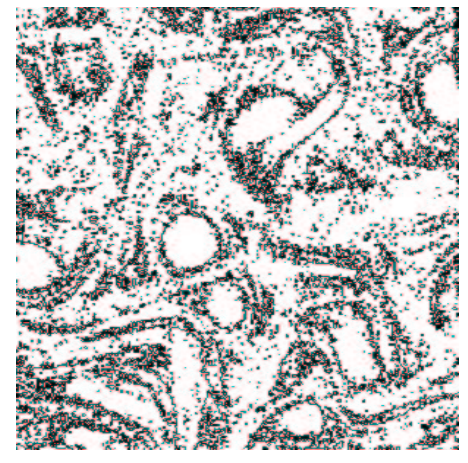

(b)

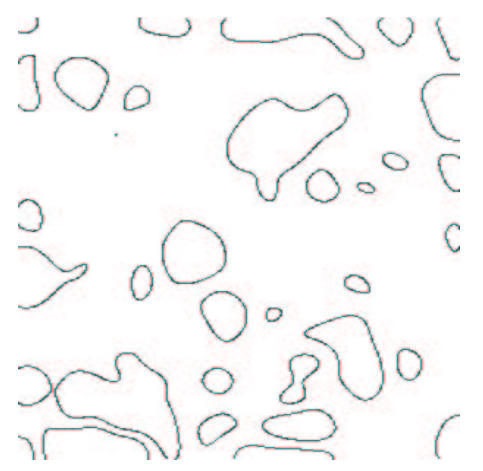

(c)

Figure 12: Vorticity contours lines (for $\mid$ curl $\mathbf{w} \mid=0.08$ ). (a) Reference contours; (b) Dense method contours; (c) Parametric estimation contours.
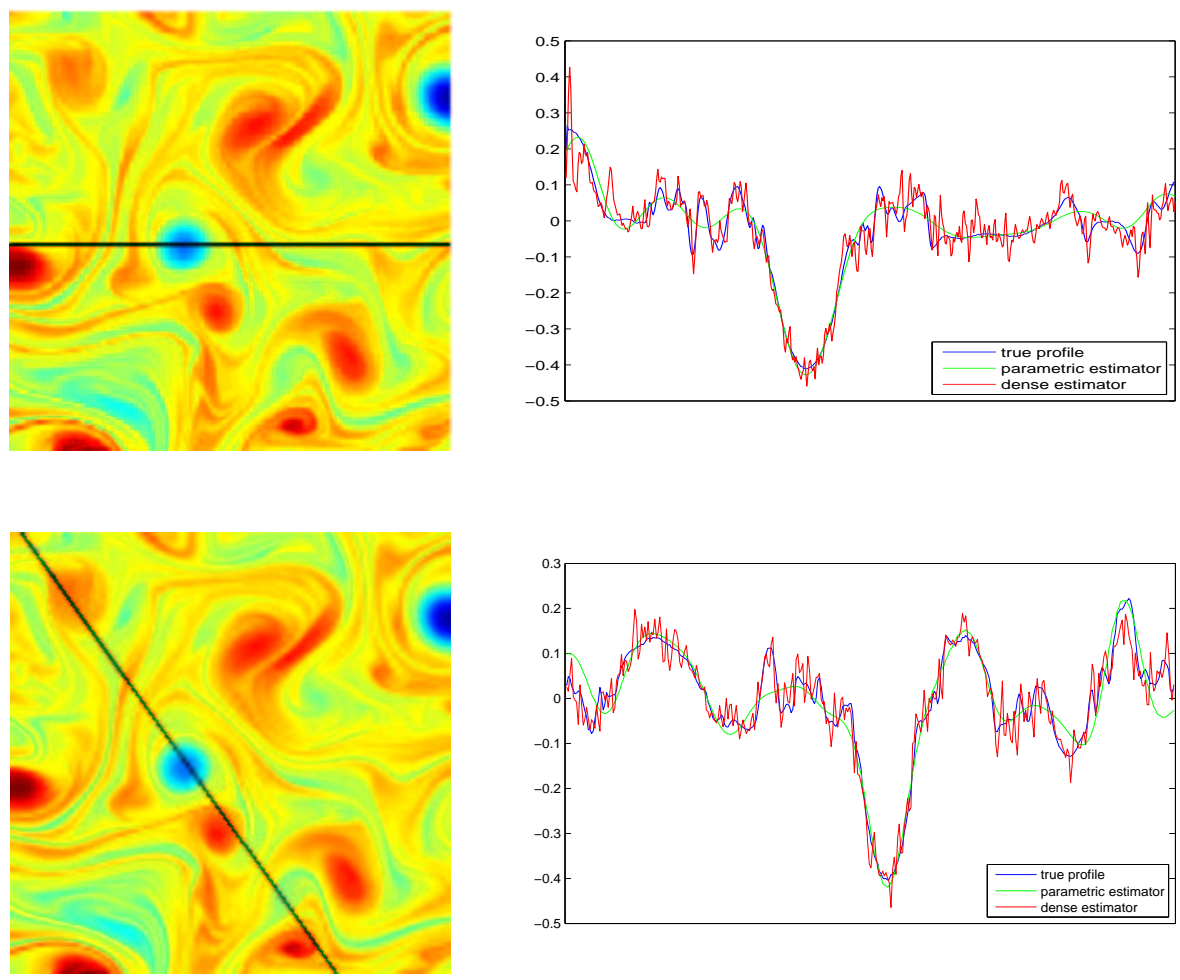

Figure 13: Vorticity profiles along two lines (represented on the left images). The green, red and blue curves show the vorticity profiles obtained respectively for the parametric estimator, the dense estimator and the actual motion field. 


\subsection{Real world sequences}

The proposed estimator has been applied on real world images of different application domains. We show in the following sections results on Meteosat meteorological images, experimental fluid mechanics images, and on medical images.

\subsubsection{Meteorological data}

Infrared channel of Meteosat The first example is a sequence of the infrared channel which shows a large depression over the Atlantic Ocean (figure 14). In this sequence we aimed at estimating the large vortex in the left part of the image. To that end 15 vortex particles have been placed manually in the vicinity of the depression. The result are presented in figure 15 . The

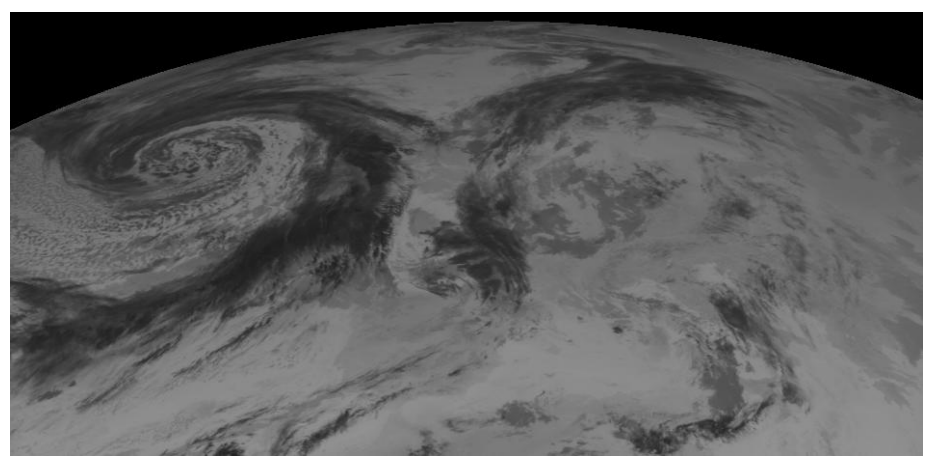

Figure 14: Meteosat image, infrared channel.

estimated transportation component is represented in figure 15 (b). The estimated solenoidal component and the underlying estimated vorticity are plotted in figures 15 (c) and 15 (d). It can be pointed out that the observed depression motion corresponds to an elongated vortex. This situation is modelized as a tripolar vortex. Such a situation emerges from 2D turbulence. 


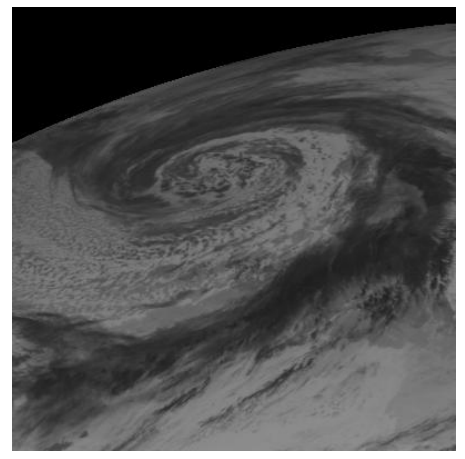

(a)

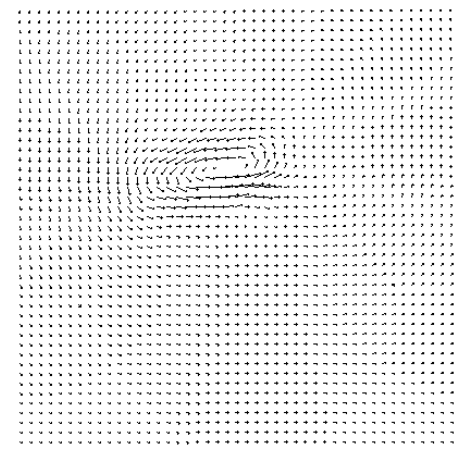

(c)

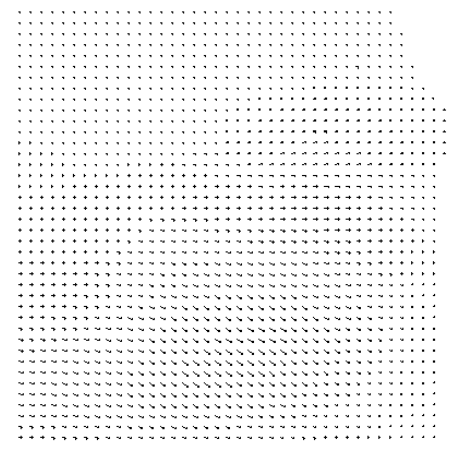

(b)

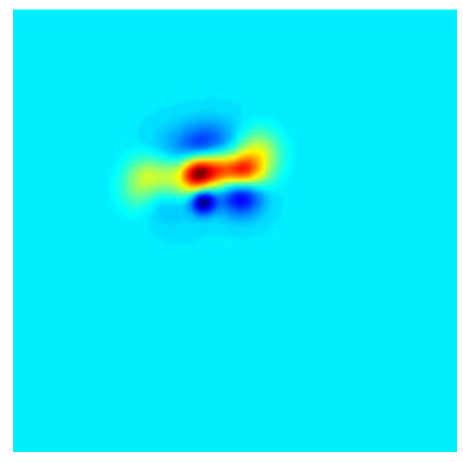

(d)

Figure 15: Result on a sequence of the infrared channel of Meteosat. (a) Image of the depression over North Atlantic; (b) Estimated transportation component; (c) Estimated solenoidal component; (d) Corresponding vorticity map.

Water vapor channel of Meteosat The second example concerns the estimation of a diverging motion from a pair of images of the water vapor channel of Meteosat. The result obtained is shown on figure 16. We can see that with only few source particles we have been able to recover the diverging motion corresponding to the apparent motion of a convective cell. Figure 17 (a) presents the initial particles locations and their estimated final positions. This example shows the efficiency of the mean shift process to estimate the optimal particles locations at the center of the divergent motion. The evolution of the divergence map estimation with the successive particles locations and their associated parameters (strength, influence) is illustrated in figure 17 (b). The extracted transportation component and the resulting diverging motion field are presented in figures $16(\mathrm{c})$ and $16(\mathrm{~d})$. 


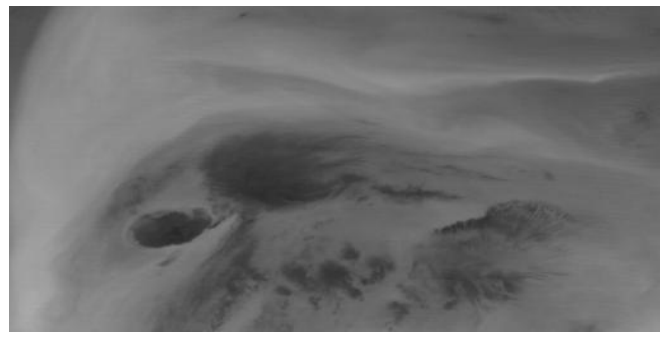

(a)

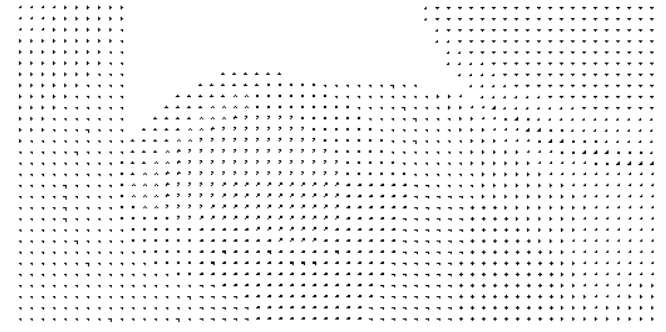

(c)

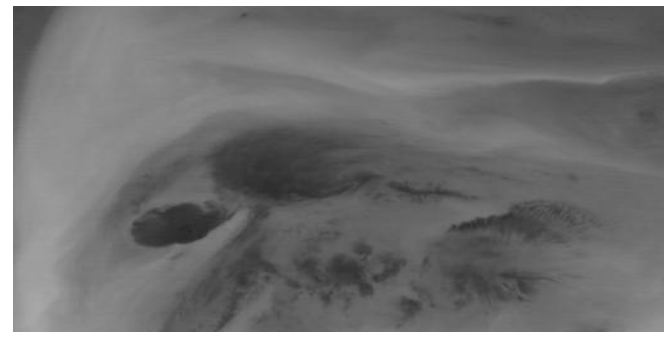

(b)

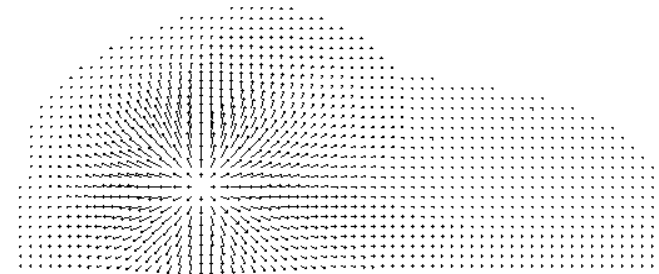

(d)

Figure 16: Result on images from the water vapor channel of Meteosat. (a) - (b) First and second images; (c) Estimated harmonic transportation component; (d) Final estimated diverging field.

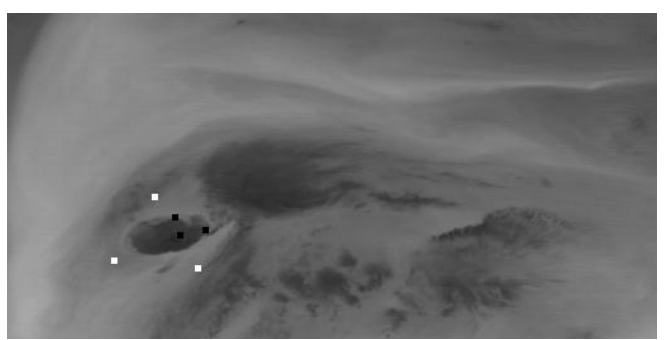

(a)

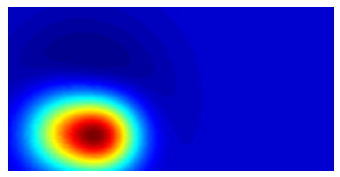

$k=0$

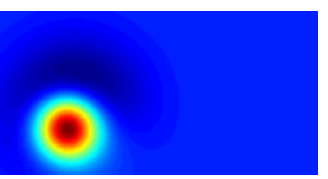

$k=1$

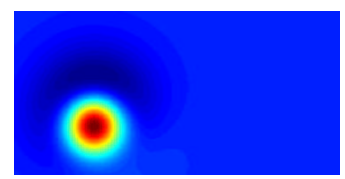

$k=2$

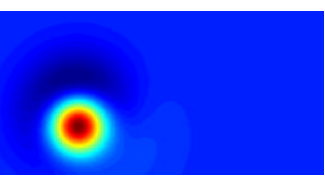

$k=3$

(b)

Figure 17: Particles locations adjustment illustration. (a) The initial particle positions are plotted on the image in white color, the final locations after convergence are plotted in black color; (b) Evolution of the estimated divergence map (convergence after three iterations). 


\subsubsection{Experimental fluid mechanics images}

The method has been experimented on images showing a big vortex launch at the tip of an airplane wing. The flow is here seeded with smoke and illuminated by a pulse laser light sheet. The figure 18 outlines the temporal consistency of the recovered solution. On this figure we have represented the recovered vorticity maps superimposed on the original smoke images, and the corresponding motion fields. As it can be observed on this figure, the method enables to recover a coherent principal vortex and a secondary vortex turning around the main one. These results have been obtained with a set of 15 vortex particles.
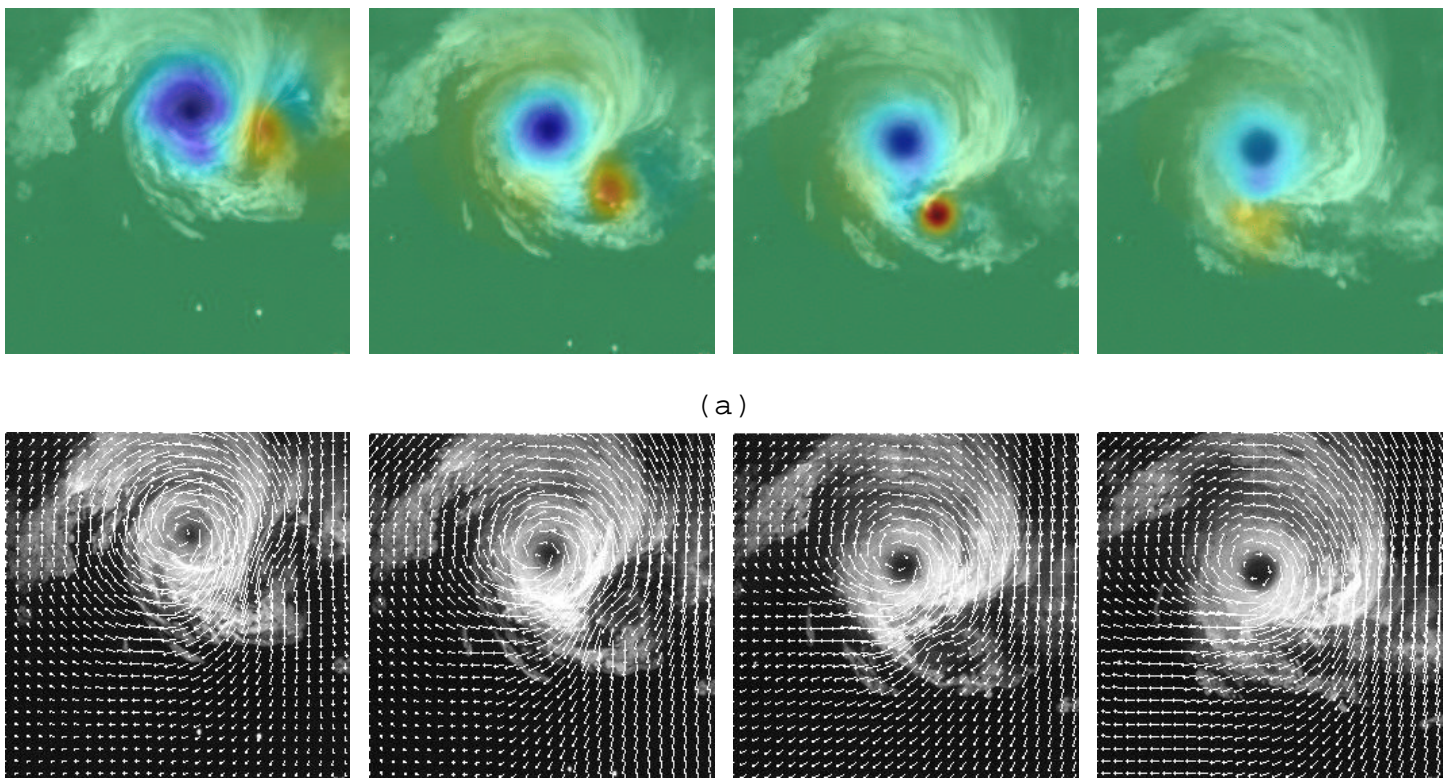

(a)
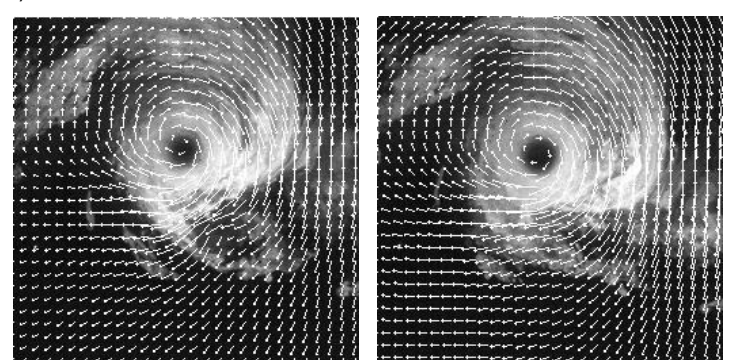

(b)

Figure 18: Vortex launch at the tip of an airplane wing (a) Vorticity maps estimated with a set of 15 vortex particles, superimposed on successive frames; (b) Estimated motion fields.

\subsubsection{Medical imaging}

The last application domain investigated in this work corresponds to a non-rigid registration issue of medical images. This is a very active field of research and numerous methods have been proposed so far. We refer the reader for comprehensive surveys on this area [30, 32, 41, 46]. This process aims at estimating a non-linear geometric transformation that puts two images into correspondence. Beyond rigid transformations, non-rigid registration is needed when deformable phenomena are observed. Application field of non-rigid registration are numerous: 
motion computation of beating organs (heart), estimation of inter-subject anatomical variability (construction of anatomical atlases), monitoring of changes over time (evolving lesions in multiple sclerosis disease), etc.

Non-rigid registration methods based on image luminance can usually be classified according to the image similarity and the deformation field regularization. Most often, methods tend to regularize deformation fields using a Gaussian regularization [40] (demon's), a first-order or second-order regularization [24] (penalization of the deformation discontinuities) or an intrinsically regularized deformation model [37] (B-splines deformation fields for instance). All these methods tend more or less to penalize the vorticity and the divergence of the motion field. This appears to be problematic when matter apparition or dissipation is observed. Such a situation undergoes a highly divergent field that has to be accurately recovered, for instance for monitoring applications of anatomical changes over time.

For this application we rely on the standard brightness consistency equation $\left(\frac{d I}{d t}=0\right)$, as the continuity equation is no longer valid in such a context. The equations of the estimation problem remain similar, except that the divergence terms are now removed.

The method has been tested on magnetic resonance images (MRI) of a patient suffering from multiple sclerosis (MS). The characterization of the lesion growth in MR images is important to assess the evolution of the disease. Two $3 D \mathrm{~T} 2$ were acquired within 6 months and rigidly registered [31]. The MR volumes have been registered with a $3 D$ rigid transformation and an axial $2 D$ slice has been extracted. The lesion appears as a white stain in the left part of the brain at $t_{0}$. Its growth is visible at $t_{0}+6$ months on figure $19(d)$.

The source particles have been initialized manually in the vicinity of the lesion. As shown on figure 20 (a) the estimation process is not very sensitive to this initialization since the particle locations adjustment process allows to guide the particles toward the region of interest. A diverging motion has been estimated, accounting for the lesion growth. The corresponding registered image is represented in figure 20 (c). The difference image between the original and the deformed image (figure $21(\mathrm{a})$ ) can be compared with the difference image after registration (figure $21(b)$ ). The error due to the lesion has been removed by the estimation method. 


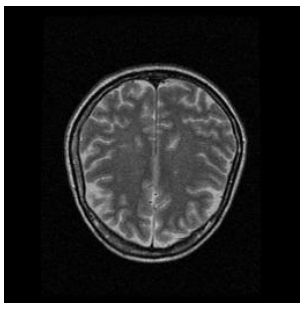

(a)

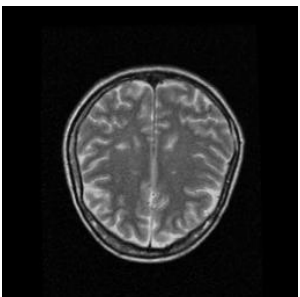

(b)

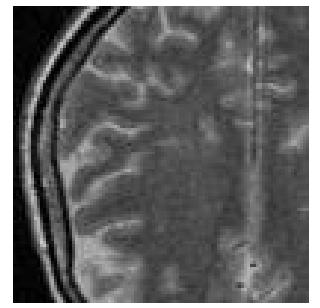

(c)

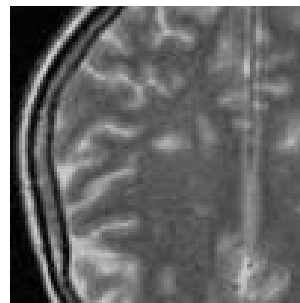

(d)

Figure 19: Evolution of a multiple sclerosis lesion (a) T2-MR slice at time $t_{0}$; (b) T2-MR corresponding slice at time $t_{0}+6$ months; $(c)-(d)$ Zoom centered on the lesion.

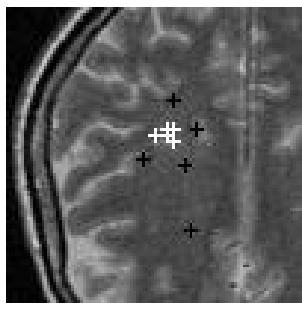

(a)

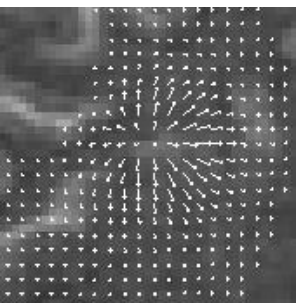

(b)

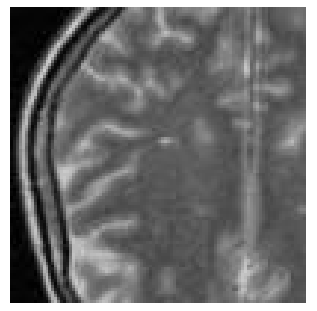

(c)

Figure 20: (a) Automatic shifting of the source particles toward the region of interest. Black points represents the initial locations of the source particles, white points the final optimal positions; (b) Zoom on the resulting divergent field, centered in the lesion; (c) Registered image (image at time $t_{0}+6$ months registered toward image at time $t_{0}$ ).

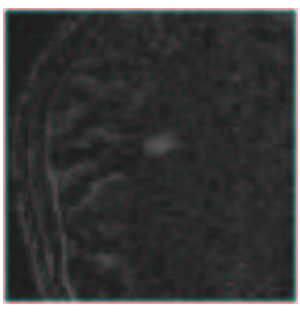

(a)

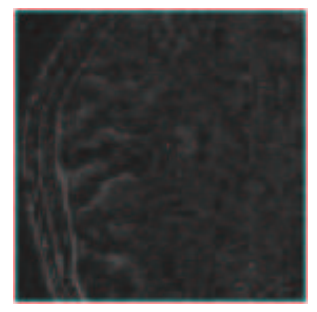

(b)

Figure 21: (a) Difference between the two images after rigid registration; (b) Difference after non-rigid registration. The lesion growth has been captured by the non-rigid registration.

\section{Remarks on computational complexity}

Before concluding let us do some remarks about the computational cost of the method. The most costly part of the estimation concerns the particles location adjustment. Considering a 
great number of particles leads to a computational cost that is higher than a dense motion estimation. Nevertheless, considering a dense fixed grid of particles leads to a reduction of the computational cost (conjugated gradient applied on the system of particles). If only a small number of particles is considered, then the estimator is quite efficient, and obviously faster than a dense estimation. Accurate measures of performance are nevertheless difficult to settle since the codes used are research codes which are not primary designed for an efficient computational objective.

\section{Conclusion}

In this paper we have presented an optical flow estimator dedicated to image sequences depicting fluid flows. The proposed estimator provides a low dimensional parametric representation of fluid motion. This parameterization has been obtained through a peculiar discretization of the divergence and the vorticity maps by means of adapted basis function centered at elements named particles. To handle the associated estimation problem, we have proposed an efficient strategy based on the coupling of a generalized conjugated gradient and a mean shift process. It must be pointed out that this method requires only few parameters. It allows as well to recover an accurate dense motion field with a great number of particles disposed over the whole image domain, or to estimate a simplified motion representation of the flow, corresponding to its large scale components. Motion estimation in targeted local areas is also authorized, which is not possible in a dense estimation context. This method dedicated to fluid flows can also be applied to deformable phenomena, in a medical context for instance. A natural extension of this work consists in a temporal tracking of the fluid flow velocity fields along the image sequence, only manageable from such a low dimensional representation of the dense motion field. The tracking can then be done within a sequential Bayesian filtering framework, with a continuous evolution law adapted to fluid flows and discrete error measurements provided by the image sequence [17].

\section{Acknowledgements}

This work was supported by the European community through the IST Fet open FLUID project (http://fluid.irisa.fr).

The authors would like to thank the ONERA for having provided them with the experimental data of wingtip vortices. 
The authors would also like to thank the CEMAGREF for the synthetic 2D turbulence data. MRI images of the MS patient are courtesy of Beatrice Carsin-Nicole (Radiology) and Gilles Edan (Neurology), Pontchaillou Hospital, Rennes, France.

\section{References}

[1] L. Alvarez, J. Weickert, and J. Sanchez. Reliable estimation of dense optical flow fields with large displacements. Int. J. Computer Vision, 39(1):41-56, 2000.

[2] A. Amini. A scalar function formulation for optical flow. In Proc. Europ. Conf. Computer Vision, pages 125-131, 1994.

[3] A. Bartoli and A. Zisserman. Direct estimation of non-rigid registrations. In Proceedings of the 15th British Machine Vision Conference, London, UK, volume II, pages 899-908, Sept 2004.

[4] D. Béréziat, I. Herlin, and L. Younes. A generalized optical flow constraint and its physical interpretation. In Proc. Conf. Comp. Vision Pattern Rec., volume 2, pages 487-492, Hilton Head Island, South Carolina, USA, 2000.

[5] M. Black and P. Anandan. The robust estimation of multiple motions: Parametric and piecewise-smooth flow fields. Computer Vision and Image Understanding, 63(1):75-104, 1996.

[6] T. Brox, A. Bruhn, N. Papenberg, and J. Weickert. High accuracy optical flow estimation based on a theory for warping. In $\operatorname{ECCV}(4)$, pages 25-36, Prague, Czech Republic, May 2004.

[7] P.J. Burt. The pyramid as a structure for efficient computation. In A. Rozenfeld, editor, Multiresolution Image Processing and Analysis, pages 6-35. Springer-Verlag, 1984.

[8] Y. Cheng. Mean shift, mode seeking and clustering. IEEE Trans. Pattern Anal. Mach. Intell., 17(8):790-799, 1995.

[9] A. J. Chorin. Numerical study of slightly viscous flow. J. Fluid Mech., 57:785-796, 1973.

[10] A.J. Chorin and J.E. Marsden. A mathematical introduction to fluid mechanics. SpringerVerlag, 1979. 
[11] D. Comaniciu and P. Meer. Mean shift: A robust approach toward feature space analysis. IEEE Trans. Pattern Analysis Machine Intelligence, 24(5):603-619, 2002.

[12] D. Comaniciu, V. Ramesh, and P. Meer. Real-time tracking of non-rigid objects using mean shift. In Proc. Conf. Comp. Vision Pattern Rec., pages 142-149, 2000.

[13] T. Corpetti, E. Mémin, and P. Pérez. Dense estimation of fluid flows. IEEE Trans. Pattern Anal. Machine Intell., 24(3):365-380, 2002.

[14] T. Corpetti, E. Mémin, and P. Pérez. Extraction of singular points from dense motion fields: an analytic approach. J. Mathematical Imaging and Vision, 19(3):175-198, 2003.

[15] G.-H. Cottet and P. Koumoutsakos. Vortex methods: theory and practice. Cambridge University Press, 2000.

[16] A. Cuzol, P. Hellier, and E. Mémin. A novel parametric method for non-rigid image registration. In G. Christensen and M. Sonka, editors, Proc. Information Processing in Medical Imaging (IPMI'05), number 3565 in LNCS, pages 456-467, Glenwood Springs, Colorado, USA, July 2005.

[17] A. Cuzol and E. Mémin. A stochastic filter for fluid motion tracking. In Proc. Int. Conf. on Computer Vision (ICCV'05), Beijing, China, October 2005.

[18] A. Cuzol and E. Mémin. Vortex and source particles for fluid motion estimation. In 5th Int. Conf. on Scale-Space and PDE methods in Computer Vision (Scale-Space'05), Hofgeismar, Germany, April 2005.

[19] J.M. Fitzpatrick. A method for calculating velocity in time dependent images based on the continuity equation. In Proc. Conf. Comp. Vision Pattern Rec., pages 78-81, San Francisco, USA, 1985.

[20] J.M. Fitzpatrick. The existence of geometrical density-image transformations corresponding to object motion. Comput. Vision, Graphics, Image Proc., 44(2):155-174, Nov. 1988.

[21] R.M. Ford, R. Strickland, and B. Thomas. Image models for 2-d flow visualization and compression. Graph. Mod. Image Proc., 56(1):75-93, 1994.

[22] K. Fukanaga and L.D. Hostetler. The estimation of the gradient of a density function, with applications in pattern recognition. IEEE Trans. on Info. Theory, 21(1):32-40, 1975. 
[23] J.C. Gilbert and J. Nocedal. Global convergence properties of conjugate gradient methods for optimization. SIAM J. Optimization, 2:21-42, 1992.

[24] P. Hellier, C. Barillot, E. Mémin, and P. Pérez. Hierarchical estimation of a dense deformation field for 3D robust registration. IEEE Transaction on Medical Imaging, 20(5):388402, 2001.

[25] B. Horn and B. Schunck. Determining optical flow. Artificial Intelligence, 17:185-203, 1981.

[26] T. Kohlberger, E. Mémin, and C. Schnörr. Variational dense motion estimation using the Helmholtz decomposition. In Int. conf on Scale-Space theories in Computer Vision(ScaleSpace '03), Isle of Skye, june 2003.

[27] E. Lamballais and J.H. Silvestrini. Direct numerical simulation of interactions between a mixing layer and a wake around a cylinder. Journal of Turbulence, 3(028), 2002.

[28] R. Larsen, K. Conradsen, and B.K. Ersboll. Estimation of dense image flow fields in fluids. IEEE trans. on Geoscience and Remote sensing, 36(1):256-264, Jan. 1998.

[29] A. Leonard. Vortex methods for flow simulation. J. Comp. Phys., 37, 1980.

[30] H. Lester and S. Arridge. A survey of hierarchical non-linear medical image registration. Pattern Recognition, 32:129-149, 1999.

[31] F. Maes, A. Collignon, D. Vandermeulen, G. Marchal, and P. Suetens. Multimodality image registration by maximisation of mutual information. IEEE Trans. Medical Imaging, 16(2):187-198, 1997.

[32] J. Maintz and MA. Viergever. A survey of medical image registration. Medical Image Analysis, 2(1):1-36, 1998.

[33] E. Mémin and P. Pérez. Dense estimation and object-based segmentation of the optical flow with robust techniques. IEEE Trans. Image Processing, 7(5):703-719, 1998.

[34] E. Mémin and P. Pérez. Fluid motion recovery by coupling dense and parametric motion fields. In Int. Conf. on Computer, ICCV'99, pages 620-625, 1999.

[35] H. Nagel and W. Enkelmann. An investigation of smoothness constraints for the estimation of displacement vector fields from image sequences. IEEE Trans. Pattern Anal. Machine Intell., 8:565-593, 1986. 
[36] A. Nomura, H. Miike, and K. Koga. Field theory approach for determining optical flow. Pattern Recognition Letters, 12(3):183-190, March 1991.

[37] D. Rueckert, I. Somoda, C. Hayes, D. Hill, M. Leach, and D. Hawkes. Nonririd registration using free-form deformations: application to breast MR images. IEEE Transactions on Medical Imaging, 18(8):712-721, 1999.

[38] B.G. Schunk. The motion constraint equation for optical flow. In Proc. Int. Conf. Pattern Recognition, pages 20-22, Montreal, 1984.

[39] S.M. Song and R.M. Leahy. Computation of $3 \mathrm{~d}$ velocity fields from $3 \mathrm{~d}$ cine and CT images of human heart. IEEE trans. on medical imaging, 10(3):295-306, 1991.

[40] J.P. Thirion. Image matching as a diffusion process: an analogy with Maxwell's demons. Medical Image Analysis, 2(3):243-260, 1998.

[41] A. Toga and P. Thompson. The role of image registration in brain mapping. Image and Vision Computing, 19:3-24, 2001.

[42] J. Weickert and C. Schnörr. Variational optic-flow computation with a spatio-temporal smoothness constraint. J. Mathematical Imaging and Vision, 14(3):245-255, 2001.

[43] R. Wildes, M. Amabile, A.M. Lanzillotto, and T.S. Leu. Physically based fluid flow recovery from image sequences. In Proc. Conf. Comp. Vision Pattern Rec., pages 969-975, 1997.

[44] J. Yuan, P. Ruhnau, E. Mémin, and C. Schnörr. Discrete orthogonal decomposition and variational fluid flow estimation. In 5th Int. Conf. on Scale-Space and PDE methods in Computer Vision (Scale-Space’05), Hofgeismar, Germany, April 2005.

[45] L. Zhou, C. Kambhamettu, and D. Goldgof. Fluid structure and motion analysis from multi-spectrum 2D cloud images sequences. In Proc. Conf. Comp. Vision Pattern Rec., volume 2, pages 744-751, Hilton Head Island, South Carolina, USA, 2000.

[46] B. Zitova and J. Flusser. Image registration methods: a survey. Image and Vision Computing, 21:977-1000, 2003. 